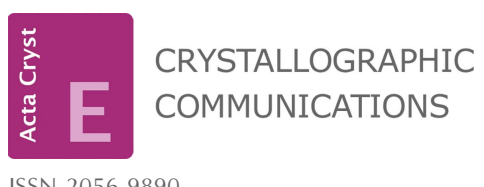

ISSN 2056-9890

Received 2 February 2021

Accepted 1 June 2021

Edited by J. T. Mague, Tulane University, USA

Keywords: crystal structure; cholesteryl; cholesterol.

CCDC reference: 2087356

Supporting information: this article has supporting information at journals.iucr.org/e

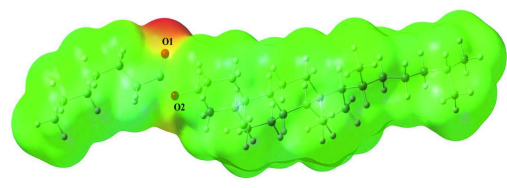

OPEN $\odot$ ACCESS

\section{Crystal structure, Hirshfeld surface analysis, interaction energy, and DFT studies of cholesteryl heptanoate}

\author{
Nurcan Akduran, ${ }^{\text {a* }}$ Tuncay Karakurt ${ }^{\mathrm{b}}$ and Tuncer Hökelek ${ }^{\mathrm{c}}$ \\ ${ }^{\mathbf{a}}$ Department of Metallurgical and Materials Engineering, Faculty of Technology, Selçuk University, 42130 Selçuklu, \\ Konya, Turkey, ${ }^{\mathbf{b}}$ Department of Chemical Engineering, Faculty of Engineering \& Architecture, Kırşehir Ahi Evran \\ University, 40100, Kırşehir, Turkey, and ' ${ }^{\mathrm{C}}$ Department of Physics, Hacettepe University, 06800 Beytepe, Ankara, Turkey. \\ *Correspondence e-mail: akduran@gmail.com
}

The title compound, $\mathrm{C}_{34} \mathrm{H}_{58} \mathrm{O}_{2}$, consists of cholesteryl and heptanoate units, in which the six-membered rings adopt chair and twisted-boat conformations while the five-membered ring adopts an envelope conformation. In the crystal, the molecules are aligned along the $a$-axis direction and stacked along the $b$-axis direction. The Hirshfeld surface analysis of the crystal structure indicates that the most important contributions for the crystal packing are from $\mathrm{H} \cdots \mathrm{H}$ $(92.4 \%)$ and $\mathrm{H} \cdots \mathrm{O} / \mathrm{O} \cdots \mathrm{H}(6.1 \%)$ interactions. van der Waals interactions are the dominant interactions in the crystal packing. Density functional theory (DFT) optimized structures at the B3LYP/ 6-31 G $(d)$ level are compared with the experimentally determined molecular structure in the solid state. The HOMO-LUMO behaviour was elucidated to determine the energy gap, and the molecular electrostatic potential (MEP) of the compound was investigated.

\section{Chemical context}

Cholesterol is an important constituent of cell membranes with a rigid ring system and a short branched hydrocarbon tail. It modulates membrane fluidity over the range of physiological temperatures and also reduces the permeability of the plasma membrane to protons and sodium ions. In the liver, it is converted to bile, which is then stored in the gallbladder. It functions in intracellular transport, cell signaling and nerve conduction within the cell membrane and is an important precursor in several biochemical pathways within the cells, in the synthesis of vitamin D and steroid hormones, including the adrenal gland hormones cortisol and aldosterone as well as sex hormones progesterone, oestrogens, and testosterone, and their derivatives. Cholesteryl esters are formed between the carboxylate group of a fatty acid and the hydroxyl group of cholesterol and have a lower solubility in water than cholesterol. These esters are also important in many biological mechanisms and numerous experimental investigations have been performed on cholesterol derivatives (Faiman et al., 1976; Goheen et al., 1977; Bush et al., 1980; Di Vizio et al., 2008; Ikonen, 2008). Thus, due to the importance of cholesterol and its esters, we report herein the crystallization, the molecular and crystal structures along with the Hirshfeld surface analysis and the interaction energy and DFT studies of the title compound, (I), whose magnetic properties were previously 
studied by electron paramagnetic resonance (EPR), (Sayin et al., 2013).

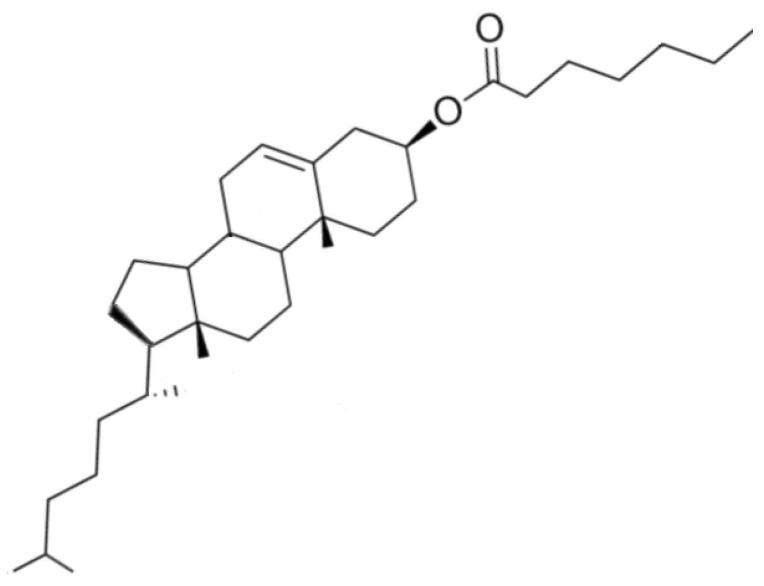

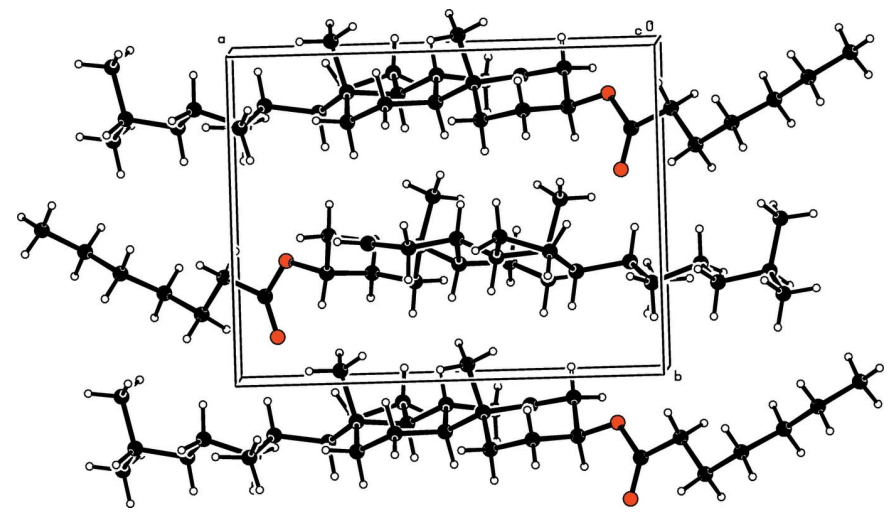

Figure 2

A partial packing diagram viewed down the $c$ axis.

\section{Supramolecular features}

In the crystal, the molecules are aligned along the $a$-axis direction and stacked along the $b$-axis direction (Fig. 2).

\section{Structural commentary}

As shown in Fig. 1, the title compound, (I), consists of cholesteryl and heptanoate units. A puckering analysis (Cremer \& Pople, 1975) of the six-membered $A$ (C8-C11/C13/ $\mathrm{C} 14), B(\mathrm{C} 10 / \mathrm{C} 11 / \mathrm{C} 15-\mathrm{C} 18), C(\mathrm{C} 17-\mathrm{C} 21 / \mathrm{C} 23)$ and the fivemembered $D(\mathrm{C} 23-\mathrm{C} 26 / \mathrm{C} 21)$ rings gave the parameters $\left[Q_{\mathrm{T}}=\right.$ $0.5403(16) \AA, \theta=6.86(18)^{\circ}$ and $\varphi=327.4(15)^{\circ}$, adopting a chair conformation (for $A$ ), $Q_{\mathrm{T}}=0.4839$ (15) $\AA, \theta=129.5(3)^{\circ}$ and $\varphi=328.2(2)^{\circ}$, adopting a twisted-boat conformation (for $B), Q_{\mathrm{T}}=0.5646(15) \AA, \theta=6.44(14)^{\circ}$ and $\varphi=245.1(14)^{\circ}$, adopting a chair conformation (for $C$ ) and $q_{2}=0.4635$ (16) $\AA$ and $\varphi=191.7(2)^{\circ}$, adopting an envelope conformation, where atom C21 is at the flap position and 0.693 (2) $\AA$ away from best plane of the remaining atoms (for $D)]$. The $\mathrm{O} 1-\mathrm{C} 7$

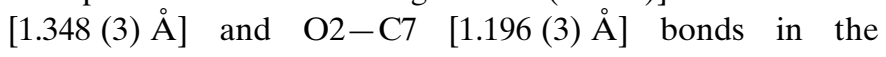
carboxylate group indicate localized single and double bonds. The $\mathrm{O} 1-\mathrm{C} 7-\mathrm{O} 2\left[123.8(2)^{\circ}\right]$ bond angle seems to be increased compared to that present in a free acid $\left[122.2^{\circ}\right]$.

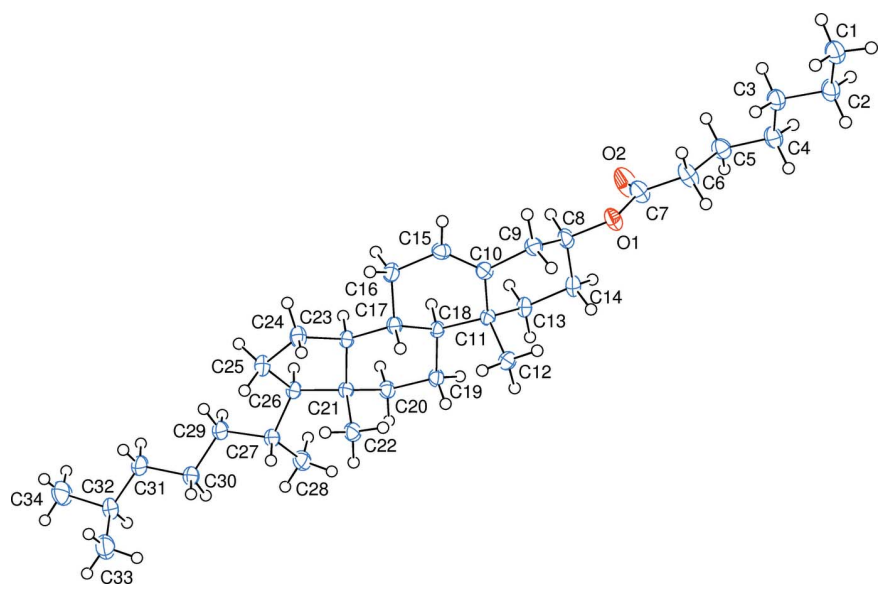

Figure 1

The asymmetric unit of the title compound with the atom-numbering scheme. Displacement ellipsoids are drawn at the $50 \%$ probability level.

\section{Hirshfeld surface analysis}

In order to visualize the intermolecular interactions in the crystal of the title compound, a Hirshfeld surface (HS) analysis (Hirshfeld, 1977; Spackman \& Jayatilaka, 2009) was carried out by using Crystal Explorer 17.5 (Turner et al., 2017). In the HS plotted over $d_{\text {norm }}$ (Fig. 3), the white surface indicates contacts with distances equal to the sum of van der Waals radii, and the red and blue colours indicate distances shorter (in close contact) or longer (distinct contact) than the van der Waals radii, respectively (Venkatesan et al., 2016). The brightred spots indicate their roles as the respective donors and/or acceptors. The overall two-dimensional fingerprint plot, Fig. $4 a$, and those delineated into $\mathrm{H} \cdots \mathrm{H}, \mathrm{H} \cdots \mathrm{O} / \mathrm{O} \cdots \mathrm{H}$ and $\mathrm{H} \cdot \mathrm{C} / \mathrm{C} \cdots \mathrm{H}$ contacts (McKinnon et al., 2007) are illustrated in Fig. $4 b-d$, respectively, together with their relative contributions to the Hirshfeld surface. The most important interaction is $\mathrm{H} \cdots \mathrm{H}$ (Table 1 ) contributing $92.4 \%$ to the overall crystal packing, which is reflected in Fig. $4 b$ as widely scattered points of high density due to the large hydrogen content of the molecule with the tip at $d_{\mathrm{e}}=d_{\mathrm{i}}=1.11 \AA$. The pair of spikes in the fingerprint plot delineated into $\mathrm{H} \cdots \mathrm{O} / \mathrm{O} \cdots \mathrm{H}$ contacts (Table 1) have a symmetrical distribution of points $(6.1 \%$ contribution, Fig. $4 c$ ) with the tips at $d_{\mathrm{e}}+d_{\mathrm{i}}=2.66 \AA$. In the absence of $\mathrm{C}-\mathrm{H} \cdots \pi$ interactions, the pair of characteristic wings in the fingerprint plot delineated into $\mathrm{H} \cdots \mathrm{C} / \mathrm{C} \cdots \mathrm{H}$

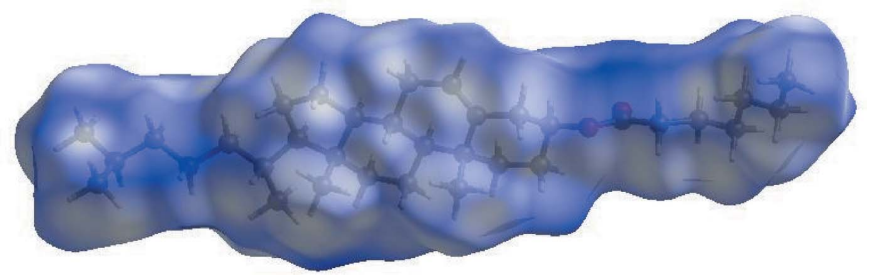

Figure 3

View of the three-dimensional Hirshfeld surface of the title compound plotted over $d_{\text {norm }}$ in the range of 0.0196 to 1.7047 a.u. 
Table 1

Selected interatomic distances $(\AA)$.

\begin{tabular}{llll}
\hline $\mathrm{O} 2 \cdots \mathrm{H} 8$ & 2.43 & $\mathrm{H} 9 A \cdots \mathrm{H} 15$ & 2.26 \\
$\mathrm{C} 9 \cdots \mathrm{H} 12 C$ & 2.78 & $\mathrm{H} 9 B \cdots \mathrm{H} 12 C$ & 2.30 \\
$\mathrm{C} 12 \cdots \mathrm{H} 19 A$ & 2.63 & $\mathrm{H} 12 A \cdots \mathrm{H} 19 A$ & 2.21 \\
$\mathrm{C} 13 \cdots \mathrm{H} 19 B$ & 2.79 & $\mathrm{H} 12 B \cdots \mathrm{H} 17$ & 2.30 \\
$\mathrm{C} 17 \cdots \mathrm{H} 22 C$ & 2.78 & $\mathrm{H} 12 C \cdots \mathrm{H} 14 A$ & 2.37 \\
$\mathrm{C} 19 \cdots \mathrm{H} 22 C$ & 2.74 & $\mathrm{H} 13 A \cdots \mathrm{H} 19 B$ & 2.29 \\
$\mathrm{C} 19 \cdots \mathrm{H} 12 A$ & 2.73 & $\mathrm{H} 13 B \cdots \mathrm{H} 18$ & 2.27 \\
$\mathrm{C} 22 \cdots \mathrm{H} 19 A$ & 2.77 & $\mathrm{H} 16 A \cdots \mathrm{H} 23$ & 2.36 \\
$\mathrm{C} 22 \cdots \mathrm{H} 27$ & 2.70 & $\mathrm{H} 17 \cdots \mathrm{H} 22 C$ & 2.26 \\
$\mathrm{C} 24 \cdots \mathrm{H} 22 B$ & 2.68 & $\mathrm{H} 19 A \cdots \mathrm{H} 22 C$ & 2.23 \\
$\mathrm{C} 25 \cdots \mathrm{H} 22 B$ & 2.71 & $\mathrm{H} 20 B \cdots \mathrm{H} 28 B$ & 2.17 \\
$\mathrm{C} 25 \cdots \mathrm{H} 29 A$ & 2.51 & $\mathrm{H} 22 B \cdots \mathrm{H} 24 B$ & 2.34 \\
$\mathrm{C} 28 \cdots \mathrm{H} 20 B$ & 2.78 & $\mathrm{H} 25 A \cdots \mathrm{H} 29 A$ & 2.32 \\
$\mathrm{C} 30 \cdots \mathrm{H} 28 A$ & 2.79 & $\mathrm{H} 28 A \cdots \mathrm{H} 30 A$ & 2.26 \\
$\mathrm{C} 30 \cdots \mathrm{H} 33 A$ & 2.75 & $\mathrm{H} 30 B \cdots \mathrm{H} 33 A$ & 2.33 \\
$\mathrm{H} 3 A \cdots \mathrm{H} 6 B$ & 2.31 & & \\
\hline
\end{tabular}

contacts (Table 1, Fig. $4 c, 1.5 \%$ contribution) has the tips at $d_{\mathrm{e}}+d_{\mathrm{i}}=2.89 \AA$.

The Hirshfeld surface representations with the function $d_{\text {norm }}$ plotted onto the surface are shown for the $\mathrm{H} \cdots \mathrm{H}$ and $\mathrm{H} \cdots \mathrm{O} / \mathrm{O} \cdots \mathrm{H}$ interactions in Fig. $5 a-b$, respectively.

The Hirshfeld surface analysis confirms the importance of $\mathrm{H}$-atom contacts in establishing the packing. The large number of $\mathrm{H} \cdots \mathrm{H}$ and $\mathrm{H} \cdots \mathrm{O} / \mathrm{O} \cdots \mathrm{H}$ interactions suggest that van der Waals interactions play the major role in the crystal packing (Hathwar et al., 2015).

\section{Interaction energy calculations}

The intermolecular interaction energies are calculated using the CE-B3LYP/6-31G(d,p) energy model available in Crystal
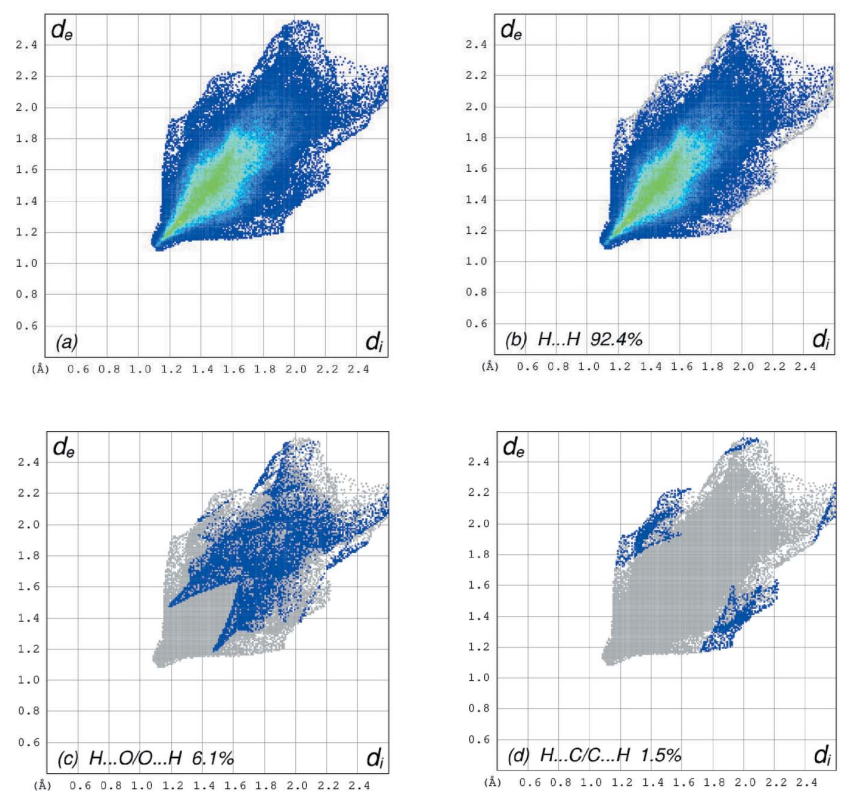

Figure 4

The full two-dimensional fingerprint plots for the title compound, showing (a) all interactions, and those delineated into $(b) \mathrm{H} \cdots \mathrm{H},(c)$ $\mathrm{H} \cdots \mathrm{O} / \mathrm{O} \cdots \mathrm{H}$ and $(d) \mathrm{H} \cdots \mathrm{C} / \mathrm{C} \cdots \mathrm{H}$ interactions. The $d_{\mathrm{i}}$ and $d_{\mathrm{e}}$ values are the closest internal and external distances (in $\AA$ ) from given points on the Hirshfeld surface contacts.
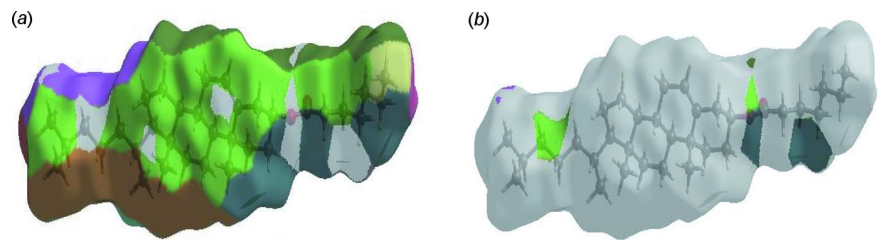

Figure 5

The Hirshfeld surface representations with the function $d_{\text {norm }}$ plotted onto the surface for $(a) \mathrm{H} \cdots \mathrm{H}$ and $(b) \mathrm{H} \cdots \mathrm{O} / \mathrm{O} \cdots \mathrm{H}$ interactions.

Explorer 17.5 (Turner et al., 2017), where a cluster of molecules is generated by applying crystallographic symmetry operations with respect to a selected central molecule within the radius of $3.8 \AA$ by default (Turner et al., 2014). The total intermolecular energy $\left(E_{\text {tot }}\right)$ is the sum of electrostatic $\left(E_{\text {ele }}\right)$, polarization $\left(E_{\mathrm{pol}}\right)$, dispersion $\left(E_{\mathrm{dis}}\right)$ and exchange-repulsion $\left(E_{\text {rep }}\right)$ energies (Turner et al., 2015) with scale factors of 1.057, 0.740, 0.871 and 0.618, respectively (Mackenzie et al., 2017). The evaluation of the energies indicates that the stabilizations in the title compound are dominated by the dispersion energy contributions.

\section{DFT calculations}

The optimized structure (Fig. 6) of the title compound was generated theoretically via density functional theory (DFT) using standard B3LYP functional and 6-31 G(d) basis-set calculations (Becke, 1993) as implemented in GAUSSIAN 09 (Frisch et al., 2009). The theoretical and experimental results were in good agreement (Table 2). As is common in these studies, there are differences between the observed and calculated values because the former pertain to the solid state while the latter are for an isolated molecule in the gas phase. The correlation graphs based on the calculations of the bond lengths and angles for comparison with the experimental results are shown in Fig. $7 a$ and $b$, respectively. The highest-

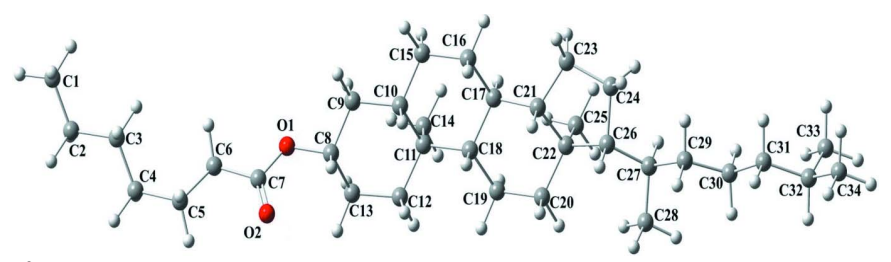

Figure 6

The optimized structure of the title compound, (I).
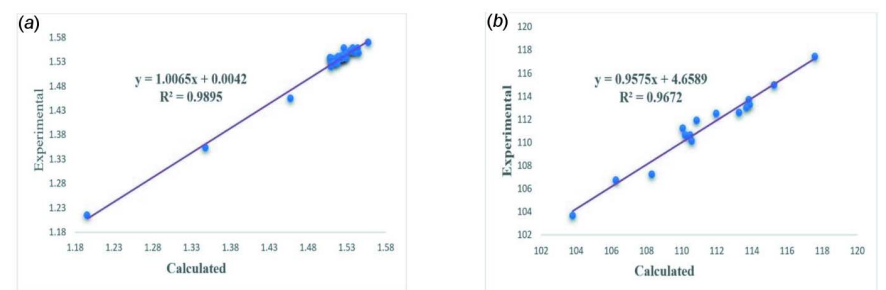

Figure 7

The correlation graphs of the calculated and experimental $(a)$ bond lengths and $(b)$ bond angles of the title compound, (I). 
Table 2

Comparison of the selected (X-ray and DFT) geometric data $\left(\AA,^{\circ}\right)$.

\begin{tabular}{|c|c|c|}
\hline Bonds/angles & X-ray & $\mathrm{B} 3 \mathrm{LYP} / 6-31 \mathrm{G}(d)$ \\
\hline $\mathrm{O} 2-\mathrm{C} 7$ & $1.196(3)$ & 1.21334 \\
\hline $\mathrm{O} 1-\mathrm{C} 7$ & $1.348(3)$ & 1.35309 \\
\hline $\mathrm{O} 1-\mathrm{C} 8$ & $1.458(2)$ & 1.45445 \\
\hline $\mathrm{C} 7-\mathrm{C} 6$ & $1.510(3)$ & 1.51813 \\
\hline $\mathrm{C} 5-\mathrm{C} 6$ & $1.516(3)$ & 1.53121 \\
\hline $\mathrm{C} 5-\mathrm{C} 4$ & $1.530(3)$ & 1.53654 \\
\hline $\mathrm{C} 4-\mathrm{C} 3$ & $1.512(4)$ & 1.53569 \\
\hline $\mathrm{C} 3-\mathrm{C} 2$ & $1.523(3)$ & 1.53425 \\
\hline $\mathrm{C} 1-\mathrm{C} 2$ & $1.510(4)$ & 1.53213 \\
\hline $\mathrm{C} 8-\mathrm{C} 14$ & $1.513(3)$ & 1.52760 \\
\hline $\mathrm{C} 8-\mathrm{C} 9$ & $1.518(3)$ & 1.52497 \\
\hline $\mathrm{C} 10-\mathrm{C} 9$ & $1.519(3)$ & 1.53951 \\
\hline $\mathrm{C} 11-\mathrm{C} 12$ & $1.545(3)$ & 1.54603 \\
\hline $\mathrm{C} 11-\mathrm{C} 18$ & $1.558(3)$ & 1.56904 \\
\hline $\mathrm{C} 17-\mathrm{C} 18$ & $1.544(3)$ & 1.55696 \\
\hline $\mathrm{C} 22-\mathrm{C} 21$ & $1.530(3)$ & 1.54490 \\
\hline $\mathrm{C} 23-\mathrm{C} 21$ & $1.538(3)$ & 1.55738 \\
\hline $\mathrm{C} 24-\mathrm{C} 23$ & $1.527(3)$ & 1.55738 \\
\hline $\mathrm{C} 24-\mathrm{C} 25$ & $1.538(3)$ & 1.55293 \\
\hline $\mathrm{C} 26-\mathrm{C} 27$ & $1.535(3)$ & 1.55117 \\
\hline $\mathrm{C} 28-\mathrm{C} 27$ & $1.528(3)$ & 1.53804 \\
\hline $\mathrm{C} 29-\mathrm{C} 27$ & $1.539(3)$ & 1.54887 \\
\hline $\mathrm{C} 29-\mathrm{C} 30$ & $1.525(3)$ & 1.53709 \\
\hline $\mathrm{C} 31-\mathrm{C} 30$ & $1.523(3)$ & 1.53617 \\
\hline $\mathrm{C} 31-\mathrm{C} 32$ & $1.524(3)$ & 1.54188 \\
\hline $\mathrm{C} 33-\mathrm{C} 32$ & $1.509(4)$ & 1.53652 \\
\hline $\mathrm{C} 34-\mathrm{C} 32$ & $1.518(4)$ & 1.53610 \\
\hline $\mathrm{C} 1-\mathrm{C} 2-\mathrm{C} 3$ & $113.9(2)$ & 113.26388 \\
\hline $\mathrm{C} 3-\mathrm{C} 4-\mathrm{C} 5$ & $115.3(2)$ & 114.95515 \\
\hline $\mathrm{C} 5-\mathrm{C} 6-\mathrm{C} 7$ & $113.7(2)$ & 112.96691 \\
\hline $\mathrm{C} 6-\mathrm{C} 7-\mathrm{O} 1$ & $110.5(2)$ & 110.59081 \\
\hline $\mathrm{C} 7-\mathrm{O} 1-\mathrm{C} 8$ & $117.58(19)$ & 117.36016 \\
\hline $\mathrm{C} 9-\mathrm{C} 8-\mathrm{C} 14$ & $110.85(19)$ & 111.83435 \\
\hline $\mathrm{C} 10-\mathrm{C} 11-\mathrm{C} 13$ & $108.31(17)$ & 107.22354 \\
\hline $\mathrm{C} 16-\mathrm{C} 17-\mathrm{C} 18$ & $110.06(17)$ & 111.14810 \\
\hline $\mathrm{C} 18-\mathrm{C} 19-\mathrm{C} 20$ & $113.82(17)$ & 113.68808 \\
\hline $\mathrm{C} 20-\mathrm{C} 21-\mathrm{C} 23$ & $106.26(17)$ & 106.65458 \\
\hline $\mathrm{C} 23-\mathrm{C} 24-\mathrm{C} 25$ & $103.79(18)$ & 103.66681 \\
\hline $\mathrm{C} 26-\mathrm{C} 27-\mathrm{C} 29$ & $110.60(18)$ & 110.09045 \\
\hline C29-C30-C31 & $112.0(2)$ & 112.44335 \\
\hline $\mathrm{C} 31-\mathrm{C} 32-\mathrm{C} 33$ & $113.3(2)$ & 112.54400 \\
\hline $\mathrm{C} 31-\mathrm{C} 32-\mathrm{C} 34$ & $110.2(2)$ & 110.56977 \\
\hline $\mathrm{C} 1-\mathrm{C} 2-\mathrm{C} 3-\mathrm{C} 4$ & $-177.7(2)$ & 179.78287 \\
\hline $\mathrm{C} 6-\mathrm{C} 7-\mathrm{O} 1-\mathrm{C} 8$ & $-179.5(2)$ & 179.67988 \\
\hline $\mathrm{C} 9-\mathrm{C} 10-\mathrm{C} 11-\mathrm{C} 18$ & $-166.45(19)$ & 164.70017 \\
\hline $\mathrm{C} 16-\mathrm{C} 17-\mathrm{C} 23-\mathrm{C} 24$ & $-57.6(3)$ & -53.53645 \\
\hline $\mathrm{C} 25-\mathrm{C} 26-\mathrm{C} 27-\mathrm{C} 29$ & $56.7(3)$ & 58.14095 \\
\hline $\mathrm{C} 29-\mathrm{C} 30-\mathrm{C} 31-\mathrm{C} 32$ & $170.8(2)$ & 174.94079 \\
\hline $\mathrm{C} 30-\mathrm{C} 31-\mathrm{C} 32-\mathrm{C} 33$ & $58.8(3)$ & 63.49014 \\
\hline $\mathrm{C} 30-\mathrm{C} 31-\mathrm{C} 32-\mathrm{C} 34$ & $-176.9(3)$ & -172.43112 \\
\hline
\end{tabular}

occupied molecular orbital (HOMO), acting as an electron donor, and the lowest-unoccupied molecular orbital (LUMO), acting as an electron acceptor, are very important parameters for quantum chemistry. When the energy gap is small, the molecule is highly polarizable and has high chemical reactivity and it is characterized as soft. The DFT calculations provide some important information on the reactivity and site selectivity of the molecular framework. $E_{\text {HOMO }}$ and $E_{\text {LUMO }}$ clarify the inevitable charge exchange collaboration inside the studied material, electronegativity $(\chi)$, hardness $(\eta)$, potential $(\mu)$, electrophilicity $(\omega)$ and softness $(\sigma)$ are recorded in Table 3. The significance of $\eta$ and $\sigma$ is to evaluate both the reactivity and stability. The HOMO and LUMO energy levels
Table 3

Calculated energies.

\begin{tabular}{lr}
\hline Molecular Energy (a.u.) $(\mathrm{eV})$ & Compound (I) \\
\hline Total Energy, $T E(\mathrm{eV})$ & -40334.80 \\
$E_{\mathrm{HOMO}}(\mathrm{eV})$ & -7.05 \\
$E_{\mathrm{LUMO}}(\mathrm{eV})$ & -0.56 \\
Gap, $\Delta E(\mathrm{eV})$ & 6.49 \\
Dipole moment, $\mu$ (Debye) & -4.07 \\
Ionization potential, $I(\mathrm{eV})$ & 7.05 \\
Electron affinity, $A$ & 0.56 \\
Electronegativity, $\chi$ & 4.06 \\
Hardness, $\eta$ & 2.14 \\
Electrophilicity index, $\omega$ & 3.85 \\
Softness, $\sigma$ & 0.23 \\
Fraction of electron transferred, $\Delta N$ & 0.49 \\
\hline
\end{tabular}

are shown in Fig. 8. The HOMO is localized in the plane extending over the whole cholesteryl heptanoate ring, while the LUMO is localized on the oxygens and their surrounding atoms. The energy band gap [ $\left.\Delta E=E_{\mathrm{LUMO}}-E_{\mathrm{HOMO}}\right]$ of the molecule is $6.49 \mathrm{eV}$, and the frontier molecular orbital energies, $E_{\mathrm{HOMO}}$ and $E_{\mathrm{LUMO}}$ are -7.05 and $-0.56 \mathrm{eV}$, respectively.

The molecular electrical potential surfaces or electrostatic potential energy maps illustrate the charge distributions of the molecules in three dimensions, allowing one to visualize variably charged regions of the molecule, which may be used to determine how molecules interact with one another. Electrostatic potential maps (MEPs) are invaluable in predicting the behaviour of complex molecules. The MEP of the title compound is shown in Fig. 9, where the negative electrostatic potential formed around $\mathrm{O} 1$ and $\mathrm{O} 2$ atoms and positive
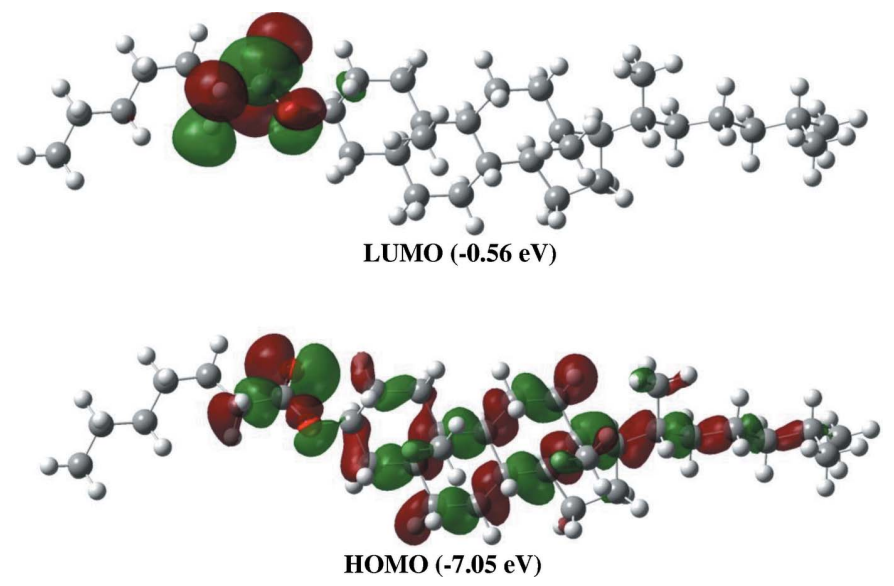

Figure 8

The LUMO and HOMO energies of the title compound, (I).

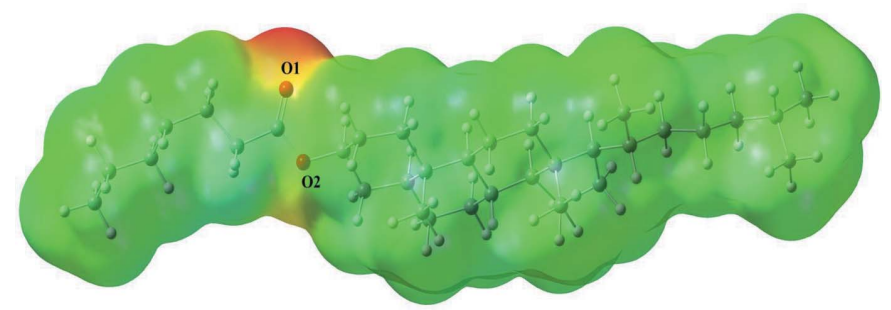

Figure 9

The MEP plot of the title compound, (I). 
Table 4

Experimental details.

\begin{tabular}{|c|c|}
\hline \multicolumn{2}{|l|}{ Crystal data } \\
\hline Chemical formula & $\mathrm{C}_{34} \mathrm{H}_{58} \mathrm{O}_{2}$ \\
\hline$M_{\mathrm{r}}$ & 498.80 \\
\hline Crystal system, space group & Monoclinic, $P 2_{1}$ \\
\hline Temperature (K) & 120 \\
\hline$a, b, c(\AA)$ & $12.0622(3), 9.2715(2), 13.8140(4)$ \\
\hline$\beta\left(^{\circ}\right)$ & $92.306(2)$ \\
\hline$V\left(\AA^{6}\right)$ & $1543.63(7)$ \\
\hline$Z$ & 2 \\
\hline Radiation type & Мo $K \alpha$ \\
\hline$\mu\left(\mathrm{mm}^{-1}\right)$ & 0.06 \\
\hline Crystal size $(\mathrm{mm})$ & $0.30 \times 0.22 \times 0.09$ \\
\hline \multicolumn{2}{|l|}{ Data collection } \\
\hline Diffractometer & $\begin{array}{l}\text { Bruker APEXII QUAZAR three- } \\
\text { circle diffractometer }\end{array}$ \\
\hline $\begin{array}{l}\text { No. of measured, independent and } \\
\text { observed }[I>2 \sigma(I)] \text { reflections }\end{array}$ & $15024,6805,6079$ \\
\hline & 0.041 \\
\hline$(\sin \theta / \lambda)_{\max }\left(\AA^{-1}\right)$ & 0.649 \\
\hline \multicolumn{2}{|l|}{ Refinement } \\
\hline$R\left[F^{2}>2 \sigma\left(F^{2}\right)\right], w R\left(F^{2}\right), S$ & $0.046,0.118,1.03$ \\
\hline No. of reflections & 6805 \\
\hline No. of parameters & 331 \\
\hline No. of restraints & 1 \\
\hline H-atom treatment & H-atom parameters constrained \\
\hline$\Delta \rho_{\max }, \Delta \rho_{\min }\left(\mathrm{e} \AA^{-3}\right)$ & $0.24,-0.22$ \\
\hline Absolute structure & $\begin{array}{l}\text { Flack } x \text { determined using } 2417 \\
\quad \text { quotients }\left[\left(I^{+}\right)-\left(I^{-}\right)\right] /\left[\left(I^{+}\right)+\left(I^{-}\right)\right] \\
\quad \text { (Parsons } \text { et al., } 2013)\end{array}$ \\
\hline Absolute structure parameter & $0.3(7)$ \\
\hline
\end{tabular}

Computer programs: APEX2 and SAINT (Bruker, 2012), SHELXT (Sheldrick, 2015a), SHELXL2018/3 (Sheldrick, 2015b), ORTEP-3 for Windows (Farrugia, 2012), WinGX publication routines (Farrugia, 2012) and PLATON (Spek, 2020).

potential (green) formed around the hydrogen atoms. The MEP values of atoms $\mathrm{O} 1$ and $\mathrm{O} 2$ are -0.050 and -0.017 a.u., respectively. Thus, atoms $\mathrm{O} 1$ and $\mathrm{O} 2$ are the most appropriate ones for electrophilic attacks while $\mathrm{H}$ atoms are more appropriate for nucleophilic attacks.

\section{Database survey}

Cholesterol and its esters take part significantly in many biological mechanisms, being important components for the manufacture of bile acids, steroid hormones and several fatsoluble vitamins. For the numerous experimental investigations, see: Faiman \& Larsson, 1976; Goheen et al., 1977; Bush et al., 1980; Di Vizio et al., 2008; Ikonen, 2008. For the first electron paramagnetic resonance (EPR) study of free radicals in X-ray-irradiated powdered cholesterol, hormones and vitamins, see: Rexroad \& Gordy, 1959. For gamma-irradiated sterol groups studied at low temperatures, see: Sevilla et al., 1986. For EPR and electron-nuclear double resonance (ENDOR) studies to elucidate the structure of free radicals formed in gamma-irradiated single crystals of selected steroids, see: Smaller \& Matheson, 1958; Krzyminiewski, Hafez et al., 1987; Krzyminiewski et al., 1990; Szyczewski \& Möbius, 1994; Szyczewski, 1996; Szyczewski et al., 1998; Çalişkan et al., 2004; Szyczewski et al., 2005; Sayin et al., 2011. For EPR studies of cholesteryl heptanoate, see: Sayin et al., 2013.

\section{Synthesis and crystallization}

The white fine crystalline powder of cholesteryl heptanoate $\left(\mathrm{C}_{34} \mathrm{H}_{58} \mathrm{O}_{2}\right)$ was purchased from Merck, and single crystals were grown by slow evaporation of a concentrated ethyl acetate solution.

\section{Refinement}

Crystal data, data collection and structure refinement details are summarized in Table 4. The $\mathrm{C}$-bound $\mathrm{H}$ atoms were positioned geometrically, with $\mathrm{C}-\mathrm{H}=0.96,0.97$ and $0.98 \AA$ for methyl, methylene and methine $\mathrm{H}$ atoms, respectively, and constrained to ride on their parent atoms, with $U_{\text {iso }}(\mathrm{H})=k \times$ $U_{\text {eq }}(\mathrm{C})$, where $k=1.5$ for methyl $\mathrm{H}$ atoms and $k=1.2$ for methylene and methine $\mathrm{H}$ atoms.

\section{Funding information}

TH is grateful to Hacettepe University Scientific Research Project Unit (grant No. 013 D04 602 004).

\section{References}

Becke, A. D. (1993). J. Chem. Phys. 98, 5648-5652.

Bruker (2012). APEX2 and SAINT. Bruker AXS Inc., Madison, Wisconsin, USA.

Bush, S. F., Levin, H. \& Levin, I. W. (1980). Chem. Phys. Lipids, 27, 101-111.

Çalişkan, B., Aras, E., Aşik, B., Büyüm, M. \& Birey, M. (2004). Radiat. Eff. Defects Solids, 159, 1-5.

Cremer, D. \& Pople, J. A. (1975). J. Am. Chem. Soc. 97, 1354-1358.

Di Vizio, D., Solomon, K. R. \& Freeman, M. R. (2008). Tumori J. 94, 633-639.

Faiman, R., Larsson, K. \& Long, D. A. (1976). J. Raman Spectrosc. 5, 3-7.

Farrugia, L. J. (2012). J. Appl. Cryst. 45, 849-854.

Frisch, M. J., Trucks, G. W., Schlegel, H. B., Scuseria, G. E., Robb, A., Cheeseman, J. R., Scalmani, G., Barone, V., Mennucci, B., Petersson, G. A., Nakatsuji, H., Caricato, M., Li, X., Hratchian, H. P., Izmaylov, A. F., Bloino, J., Zheng, G., Sonnenberg, J. L., Hada, M., Ehara, M., Toyota, K., Fukuda, R., Hasegawa, J., Ishida, M., Nakajima, T., Honda, Y., Kitao, O., Nakai, H., Vreven, T., Montgomery, J. A. Jr, Peralta, J. E., Ogliaro, F., Bearpark, M., Heyd, J. J., Brothers, E., Kudin, K. N., Staroverov, V. N., Kobayashi, R., Normand, J., Raghavachari, K., Rendell, A., Burant, J. C., Iyengar, S. S., Tomasi, J., Cossi, M., Rega, N., Millam, J. M., Klene, M., Knox, J. E., Cross, J. B., Bakken, V., Adamo, C., Jaramillo, J., Gomperts, R., Stratmann, R. E., Yazyev, O., Austin, A. J., Cammi, R., Pomelli, C., Ochterski, J. W., Martin, R. L., Morokuma, K., Zakrzewski, V. G., Voth, G. A., Salvador, P., Dannenberg, J. J., Dapprich, S., Daniels, A. D., Farkas, Ö., Foresman, J. B., Ortiz, J. V., Cioslowski, J. \& Fox, D. J. (2009). GAUSSIAN09. Gaussian Inc., Wallingford, CT, USA.

Goheen, S. C., Lis, L. J. \& Kauffman, J. W. (1977). Chem. Phys. Lipids, 20, 253-262.

Hathwar, V. R., Sist, M., Jørgensen, M. R. V., Mamakhel, A. H., Wang, X., Hoffmann, C. M., Sugimoto, K., Overgaard, J. \& Iversen, B. B. (2015). IUCrJ, 2, 563-574.

Hirshfeld, H. L. (1977). Theor. Chim. Acta, 44, 129-138.

Ikonen, E. (2008). Nat. Rev. Mol. Cell Biol. 9, 125-138.

Krzyminiewski, R., Hafez, A. M., Szyczewski, A. \& Pietrzak, J. (1987). J. Mol. Struct. 160, 127-133.

Krzyminiewski, R., Pietrzak, J. \& Konopka, R. (1990). J. Mol. Struct. 240, 133-140. 
Mackenzie, C. F., Spackman, P. R., Jayatilaka, D. \& Spackman, M. A. (2017). IUCrJ, 4, 575-587.

McKinnon, J. J., Jayatilaka, D. \& Spackman, M. A. (2007). Chem. Commun. pp. 3814-3816.

Parsons, S., Flack, H. D. \& Wagner, T. (2013). Acta Cryst. B69, 249259.

Rexroad, H. N. \& Gordy, W. (1959). Proc. Natl Acad. Sci. USA, 45, 256-269.

Sayin, U., Can, C., Türkkan, E., Dereli, Ö., Ozmen, A. \& Yüksel, H. (2013). Acta Phys. Pol. A, 124, 70-73.

Sayin, U., Yüksel, H. \& Birey, M. (2011). Radiat. Phys. Chem. 80, 1203-1207.

Sevilla, C. L., Becker, D. \& Sevilla, M. D. (1986). J. Phys. Chem. 90, 2963-2968.

Sheldrick, G. M. (2015a). Acta Cryst. A71, 3-8.

Sheldrick, G. M. (2015b). Acta Cryst. C71, 3-8.

Smaller, B. \& Matheson, M. S. (1958). J. Chem. Phys. 28, 1169-1178.
Spackman, M. A. \& Jayatilaka, D. (2009). CrystEngComm, 11, 19-32. Spek, A. L. (2020). Acta Cryst. E76, 1-11.

Szyczewski, A. (1996). Appl. Radiat. Isot. 47, 1675-1681.

Szyczewski, A., Endeward, B. \& Möbius, K. (1998). Appl. Radiat. Isot. 49, 59-65.

Szyczewski, A. \& Möbius, K. (1994). J. Mol. Struct. 318, 87-93.

Szyczewski, A., Pietrzak, J. \& Möbius, K. (2005). Acta Phys. Pol. A, 108, 119-126.

Turner, M. J., Grabowsky, S., Jayatilaka, D. \& Spackman, M. A. (2014). J. Phys. Chem. Lett. 5, 4249-4255.

Turner, M. J., McKinnon, J. J., Wolff, S. K., Grimwood, D. J., Spackman, P. R., Jayatilaka, D. \& Spackman, M. A. (2017). CrystalExplorer17. The University of Western Australia.

Turner, M. J., Thomas, S. P., Shi, M. W., Jayatilaka, D. \& Spackman, M. A. (2015). Chem. Commun. 51, 3735-3738.

Venkatesan, P., Thamotharan, S., Ilangovan, A., Liang, H. \& Sundius, T. (2016). Spectrochim. Acta Part A, 153, 625-636. 


\section{supporting information}

Acta Cryst. (2021). E77, 686-691［https://doi.org/10.1107/S2056989021005661]

\section{Crystal structure, Hirshfeld surface analysis, interaction energy, and DFT studies of cholesteryl heptanoate}

\section{Nurcan Akduran, Tuncay Karakurt and Tuncer Hökelek}

Computing details

Data collection: APEX2 (Bruker, 2012); cell refinement: SAINT (Bruker, 2012); data reduction: SAINT (Bruker, 2012); program(s) used to solve structure: SHELXT (Sheldrick, 2015a); program(s) used to refine structure: SHELXL2018/3 (Sheldrick, 2015b); molecular graphics: ORTEP-3 for Windows (Farrugia, 2012); software used to prepare material for publication: Win GX publication routines (Farrugia, 2012) and PLATON (Spek, 2020).

Cholesteryl heptanoate

Crystal data

$\mathrm{C}_{34} \mathrm{H}_{58} \mathrm{O}_{2}$

$M_{r}=498.80$

Monoclinic, $P 2_{1}$

$a=12.0622(3) \AA$

$b=9.2715$ (2) $\AA$

$c=13.8140$ (4) $\AA$

$\beta=92.306(2)^{\circ}$

$V=1543.63(7) \AA^{3}$

$Z=2$

\section{Data collection}

Bruker APEXII QUAZAR three-circle diffractometer

Detector resolution: 8.3333 pixels $\mathrm{mm}^{-1}$ $\varphi$ and $\omega$ scans 15024 measured reflections 6805 independent reflections

\section{Refinement}

Refinement on $F^{2}$

Least-squares matrix: full

$R\left[F^{2}>2 \sigma\left(F^{2}\right)\right]=0.046$

$w R\left(F^{2}\right)=0.118$

$S=1.03$

6805 reflections

331 parameters

1 restraint

Hydrogen site location: inferred from neighbouring sites
$F(000)=556$

$D_{\mathrm{x}}=1.073 \mathrm{Mg} \mathrm{m}^{-3}$

Mo $K \alpha$ radiation, $\lambda=0.71073 \AA$

Cell parameters from 5761 reflections

$\theta=2.2-27.3^{\circ}$

$\mu=0.06 \mathrm{~mm}^{-1}$

$T=120 \mathrm{~K}$

Plate, colourless

$0.30 \times 0.22 \times 0.09 \mathrm{~mm}$

6079 reflections with $I>2 \sigma(I)$

$R_{\text {int }}=0.041$

$\theta_{\max }=27.5^{\circ}, \theta_{\min }=1.5^{\circ}$

$h=-15 \rightarrow 15$

$k=-12 \rightarrow 12$

$l=-17 \rightarrow 17$

$\mathrm{H}$-atom parameters constrained

$w=1 /\left[\sigma^{2}\left(F_{\mathrm{o}}^{2}\right)+(0.0614 P)^{2}+0.1758 P\right]$ where $P=\left(F_{\mathrm{o}}^{2}+2 F_{\mathrm{c}}^{2}\right) / 3$

$(\Delta / \sigma)_{\max }<0.001$

$\Delta \rho_{\max }=0.24 \mathrm{e} \AA^{-3}$

$\Delta \rho_{\min }=-0.22$ e $\AA^{-3}$

Absolute structure: Flack $x$ determined using 2417 quotients $\left[\left(I^{+}\right)-\left(I^{-}\right)\right] /\left[\left(I^{+}\right)+\left(I^{-}\right)\right]$(Parsons et al., 2013)

Absolute structure parameter: 0.3 (7) 


\section{Special details}

Geometry. All esds (except the esd in the dihedral angle between two 1.s. planes) are estimated using the full covariance matrix. The cell esds are taken into account individually in the estimation of esds in distances, angles and torsion angles; correlations between esds in cell parameters are only used when they are defined by crystal symmetry. An approximate (isotropic) treatment of cell esds is used for estimating esds involving l.s. planes.

Fractional atomic coordinates and isotropic or equivalent isotropic displacement parameters $\left(\AA^{2}\right)$

\begin{tabular}{|c|c|c|c|c|}
\hline & $x$ & $y$ & $z$ & $U_{\text {iso }} * / U_{\text {eq }}$ \\
\hline $\mathrm{O} 1$ & $0.88629(14)$ & $0.64631(18)$ & $0.15841(13)$ & $0.0314(4)$ \\
\hline $\mathrm{O} 2$ & $0.91179(18)$ & $0.8775(2)$ & $0.11617(18)$ & $0.0518(6)$ \\
\hline $\mathrm{C} 1$ & $1.4569(2)$ & $0.5375(3)$ & $-0.03304(19)$ & $0.0381(6)$ \\
\hline $\mathrm{H} 1 \mathrm{~A}$ & 1.504609 & 0.496801 & -0.079854 & $0.057^{*}$ \\
\hline H1B & 1.431548 & 0.462591 & 0.008619 & $0.057^{*}$ \\
\hline $\mathrm{H} 1 \mathrm{C}$ & 1.497197 & 0.608290 & 0.004961 & $0.057^{*}$ \\
\hline $\mathrm{C} 2$ & $1.3584(2)$ & $0.6081(3)$ & $-0.08471(17)$ & $0.0316(5)$ \\
\hline $\mathrm{H} 2 \mathrm{~A}$ & 1.320002 & 0.536527 & -0.124748 & $0.038^{*}$ \\
\hline $\mathrm{H} 2 \mathrm{~B}$ & 1.385067 & 0.682562 & -0.127298 & $0.038^{*}$ \\
\hline $\mathrm{C} 3$ & $1.2763(2)$ & $0.6752(3)$ & $-0.01697(17)$ & $0.0330(6)$ \\
\hline $\mathrm{H} 3 \mathrm{~A}$ & 1.251610 & 0.601733 & 0.027306 & $0.040^{*}$ \\
\hline H3B & 1.313740 & 0.749700 & 0.021227 & $0.040^{*}$ \\
\hline $\mathrm{C} 4$ & $1.1761(2)$ & $0.7401(3)$ & $-0.06989(18)$ & $0.0308(5)$ \\
\hline $\mathrm{H} 4 \mathrm{~A}$ & 1.140592 & 0.665786 & -0.109617 & $0.037^{*}$ \\
\hline H4B & 1.201347 & 0.814656 & -0.113085 & $0.037^{*}$ \\
\hline $\mathrm{C} 5$ & $1.0894(2)$ & $0.8056(3)$ & -0.00503 (19) & $0.0304(5)$ \\
\hline $\mathrm{H} 5 \mathrm{~A}$ & 1.124902 & 0.877278 & 0.036917 & $0.036^{*}$ \\
\hline H5B & 1.033294 & 0.854229 & -0.045343 & $0.036^{*}$ \\
\hline C6 & $1.0337(2)$ & $0.6940(3)$ & $0.0568(2)$ & $0.0367(6)$ \\
\hline H6A & 1.006225 & 0.616313 & 0.015417 & $0.044^{*}$ \\
\hline H6B & 1.088515 & 0.653667 & 0.102419 & $0.044^{*}$ \\
\hline $\mathrm{C} 7$ & $0.9385(2)$ & $0.7534(3)$ & $0.1125(2)$ & $0.0335(6)$ \\
\hline $\mathrm{C} 8$ & $0.79138(19)$ & $0.6851(3)$ & $0.21509(17)$ & $0.0273(5)$ \\
\hline $\mathrm{H} 8$ & 0.804295 & 0.779292 & 0.245756 & $0.033^{*}$ \\
\hline $\mathrm{C} 9$ & $0.78323(19)$ & $0.5697(3)$ & $0.29241(17)$ & $0.0261(5)$ \\
\hline H9A & 0.848991 & 0.572960 & 0.335142 & $0.031 *$ \\
\hline H9B & 0.780098 & 0.475481 & 0.261901 & $0.031^{*}$ \\
\hline $\mathrm{C} 10$ & $0.68089(18)$ & $0.5910(2)$ & $0.35138(16)$ & $0.0220(4)$ \\
\hline $\mathrm{C} 11$ & $0.57079(18)$ & $0.6073(2)$ & $0.29501(15)$ & $0.0205(4)$ \\
\hline $\mathrm{C} 12$ & $0.5379(2)$ & $0.4598(3)$ & $0.25045(17)$ & $0.0267(5)$ \\
\hline $\mathrm{H} 12 \mathrm{~A}$ & 0.475360 & 0.471934 & 0.206043 & $0.040^{*}$ \\
\hline H12B & 0.518810 & 0.394491 & 0.301058 & $0.040^{*}$ \\
\hline $\mathrm{H} 12 \mathrm{C}$ & 0.599259 & 0.421289 & 0.216544 & $0.040^{*}$ \\
\hline $\mathrm{C} 13$ & $0.58615(19)$ & $0.7181(3)$ & $0.21267(16)$ & $0.0247(5)$ \\
\hline H13A & 0.519863 & 0.717802 & 0.170516 & $0.030^{*}$ \\
\hline H13B & 0.593196 & 0.813491 & 0.241059 & $0.030^{*}$ \\
\hline $\mathrm{C} 14$ & 0.68645 (19) & $0.6904(3)$ & $0.15136(17)$ & $0.0278(5)$ \\
\hline H14A & 0.676936 & 0.599652 & 0.117096 & $0.033^{*}$ \\
\hline H14B & 0.692363 & 0.766606 & 0.103719 & $0.033^{*}$ \\
\hline
\end{tabular}




\begin{tabular}{|c|c|c|c|c|}
\hline $\mathrm{C} 15$ & $0.69011(18)$ & $0.5926(2)$ & $0.44767(16)$ & $0.0242(5)$ \\
\hline H15 & 0.760872 & 0.583827 & 0.476350 & $0.029 *$ \\
\hline $\mathrm{C} 16$ & $0.59494(18)$ & $0.6076(3)$ & $0.51323(15)$ & $0.0249(5)$ \\
\hline H16A & 0.596925 & 0.703036 & 0.542050 & $0.030^{*}$ \\
\hline H16B & 0.603350 & 0.537672 & 0.565224 & $0.030 *$ \\
\hline $\mathrm{C} 17$ & $0.48277(18)$ & $0.5853(2)$ & $0.46075(15)$ & 0.0205 (4) \\
\hline H17 & 0.471506 & 0.481913 & 0.449347 & $0.025^{*}$ \\
\hline $\mathrm{C} 18$ & $0.47990(17)$ & $0.6643(2)$ & $0.36225(15)$ & $0.0195(4)$ \\
\hline H18 & 0.498642 & 0.765154 & 0.376379 & $0.023 *$ \\
\hline $\mathrm{C} 19$ & $0.36285(18)$ & $0.6654(3)$ & $0.31454(15)$ & $0.0241(5)$ \\
\hline H19A & 0.344502 & 0.568507 & 0.292694 & $0.029^{*}$ \\
\hline H19B & 0.362694 & 0.727231 & 0.257918 & $0.029^{*}$ \\
\hline $\mathrm{C} 20$ & $0.27255(18)$ & 0.7178 & $0.38188(16)$ & $0.0237(5)$ \\
\hline $\mathrm{H} 20 \mathrm{~A}$ & 0.285173 & 0.818564 & 0.397559 & $0.028^{*}$ \\
\hline $\mathrm{H} 20 \mathrm{~B}$ & 0.200528 & 0.709896 & 0.348451 & $0.028^{*}$ \\
\hline $\mathrm{C} 21$ & $0.27233(17)$ & $0.6294(2)$ & $0.47595(15)$ & 0.0199 (4) \\
\hline $\mathrm{C} 22$ & $0.2384(2)$ & $0.4731(2)$ & $0.45499(18)$ & 0.0269 \\
\hline $\mathrm{H} 22 \mathrm{~A}$ & 0.166143 & 0.471404 & 0.423254 & $0.040^{*}$ \\
\hline $\mathrm{H} 22 \mathrm{~B}$ & 0.236660 & 0.420533 & 0.514769 & $0.040^{*}$ \\
\hline $\mathrm{H} 22 \mathrm{C}$ & 0.291140 & 0.429430 & 0.413806 & $0.040^{*}$ \\
\hline $\mathrm{C} 23$ & $0.39003(17)$ & $0.6415(2)$ & $0.52227(14)$ & $0.0196(4)$ \\
\hline $\mathrm{H} 23$ & 0.404155 & 0.744866 & 0.531215 & $0.024 *$ \\
\hline $\mathrm{C} 24$ & $0.3794(2)$ & 0.5788 & $0.62369(16)$ & $0.0272(5)$ \\
\hline $\mathrm{H} 24 \mathrm{~A}$ & 0.436235 & 0.617339 & 0.668192 & $0.033^{*}$ \\
\hline $\mathrm{H} 24 \mathrm{~B}$ & 0.385290 & 0.474515 & 0.622739 & $0.033^{*}$ \\
\hline $\mathrm{C} 25$ & $0.26318(19)$ & $0.6266(3)$ & $0.65246(16)$ & $0.0266(5)$ \\
\hline $\mathrm{H} 25 \mathrm{~A}$ & 0.268795 & 0.698477 & 0.703457 & $0.032 *$ \\
\hline H25B & 0.221731 & 0.544824 & 0.675685 & $0.032 *$ \\
\hline $\mathrm{C} 26$ & $0.20397(17)$ & $0.6915(2)$ & $0.55966(15)$ & $0.0210(4)$ \\
\hline $\mathrm{H} 26$ & 0.216305 & 0.795963 & 0.561267 & $0.025^{*}$ \\
\hline $\mathrm{C} 27$ & $0.07807(18)$ & $0.6667(3)$ & $0.55763(15)$ & $0.0251(5)$ \\
\hline $\mathrm{H} 27$ & 0.065026 & 0.562329 & 0.557622 & $0.030^{*}$ \\
\hline $\mathrm{C} 28$ & $0.0200(2)$ & 0.7293 & $0.46669(18)$ & $0.0342(6)$ \\
\hline $\mathrm{H} 28 \mathrm{~A}$ & -0.058932 & 0.724501 & 0.472814 & $0.051^{*}$ \\
\hline $\mathrm{H} 28 \mathrm{~B}$ & 0.040410 & 0.674760 & 0.411099 & $0.051^{*}$ \\
\hline $\mathrm{H} 28 \mathrm{C}$ & 0.041967 & 0.828039 & 0.459043 & $0.051^{*}$ \\
\hline $\mathrm{C} 29$ & $0.02759(19)$ & 0.7298 & $0.64897(17)$ & $0.0295(5)$ \\
\hline $\mathrm{H} 29 \mathrm{~A}$ & 0.076544 & 0.707596 & 0.704403 & $0.035^{*}$ \\
\hline H29B & 0.024675 & 0.833916 & 0.642601 & $0.035^{*}$ \\
\hline $\mathrm{C} 30$ & $-0.08842(19)$ & 0.6749 & $0.66924(16)$ & $0.0274(5)$ \\
\hline $\mathrm{H} 30 \mathrm{~A}$ & -0.139634 & 0.705361 & 0.617273 & $0.033^{*}$ \\
\hline Н30B & -0.087729 & 0.570343 & 0.670378 & $0.033^{*}$ \\
\hline $\mathrm{C} 31$ & $-0.1289(2)$ & $0.7309(3)$ & $0.76532(18)$ & $0.0355(6)$ \\
\hline H31A & -0.117039 & 0.834280 & 0.767913 & $0.043^{*}$ \\
\hline H31B & -0.083762 & 0.687938 & 0.817400 & $0.043^{*}$ \\
\hline $\mathrm{C} 32$ & $-0.2505(2)$ & $0.7007(3)$ & $0.78366(17)$ & $0.0302(5)$ \\
\hline $\mathrm{H} 32$ & -0.295137 & 0.747125 & 0.731650 & $0.036^{*}$ \\
\hline $\mathrm{C} 33$ & $-0.2788(3)$ & $0.5422(3)$ & $0.7824(2)$ & $0.0433(7)$ \\
\hline
\end{tabular}




$\begin{array}{lllll}\text { H33A } & -0.262037 & 0.501912 & 0.720684 & 0.065^{*} \\ \text { H33B } & -0.356401 & 0.530157 & 0.793139 & 0.065^{*} \\ \text { H33C } & -0.236003 & 0.493674 & 0.832650 & 0.065^{*} \\ \text { C34 } & -0.2821(3) & 0.7685(4) & 0.8787(2) & 0.0544(9) \\ \text { H34A } & -0.266938 & 0.870126 & 0.877199 & 0.082^{*} \\ \text { H34B } & -0.239742 & 0.724853 & 0.931153 & 0.082^{*} \\ \text { H34C } & -0.359790 & 0.753511 & 0.887713 & 0.082^{*}\end{array}$

Atomic displacement parameters $\left(A^{2}\right)$

\begin{tabular}{|c|c|c|c|c|c|c|}
\hline & $U^{11}$ & $U^{22}$ & $U^{33}$ & $U^{12}$ & $U^{13}$ & $U^{23}$ \\
\hline $\mathrm{O} 1$ & $0.0290(9)$ & $0.0269(9)$ & $0.0393(10)$ & $0.0008(7)$ & $0.0154(7)$ & $-0.0006(7)$ \\
\hline $\mathrm{O} 2$ & $0.0526(13)$ & $0.0282(10)$ & $0.0772(16)$ & $0.0055(9)$ & $0.0329(11)$ & $0.0059(10)$ \\
\hline $\mathrm{C} 1$ & $0.0320(14)$ & $0.0523(17)$ & $0.0305(13)$ & $-0.0027(12)$ & $0.0073(11)$ & $0.0017(12)$ \\
\hline $\mathrm{C} 2$ & $0.0344(13)$ & $0.0339(13)$ & $0.0269(12)$ & $-0.0017(11)$ & $0.0082(10)$ & $0.0022(10)$ \\
\hline $\mathrm{C} 3$ & $0.0293(12)$ & $0.0445(15)$ & $0.0253(11)$ & $-0.0018(11)$ & $0.0048(9)$ & $0.0024(11)$ \\
\hline $\mathrm{C} 4$ & $0.0330(13)$ & $0.0312(13)$ & $0.0287(12)$ & $-0.0034(10)$ & $0.0087(10)$ & $0.0065(10)$ \\
\hline $\mathrm{C} 5$ & $0.0315(13)$ & $0.0260(12)$ & $0.0339(13)$ & $-0.0021(10)$ & $0.0053(10)$ & $0.0026(10)$ \\
\hline C6 & $0.0329(13)$ & $0.0294(13)$ & $0.0492(15)$ & $0.0020(11)$ & $0.0183(11)$ & $0.0053(12)$ \\
\hline $\mathrm{C} 7$ & $0.0314(13)$ & $0.0293(13)$ & $0.0406(14)$ & $-0.0007(10)$ & $0.0104(11)$ & $0.0020(11)$ \\
\hline $\mathrm{C} 8$ & $0.0262(11)$ & $0.0255(11)$ & $0.0309(12)$ & $0.0001(10)$ & $0.0114(9)$ & $-0.0057(10)$ \\
\hline C9 & $0.0222(11)$ & $0.0281(12)$ & $0.0281(11)$ & $0.0011(9)$ & $0.0028(9)$ & $-0.0035(9)$ \\
\hline $\mathrm{C} 10$ & $0.0224(11)$ & $0.0172(10)$ & $0.0265(11)$ & $0.0008(8)$ & $0.0039(8)$ & $-0.0032(8)$ \\
\hline $\mathrm{C} 11$ & $0.0219(10)$ & $0.0204(11)$ & $0.0195(10)$ & $-0.0006(8)$ & $0.0029(8)$ & $-0.0028(8)$ \\
\hline $\mathrm{C} 12$ & $0.0285(12)$ & $0.0244(11)$ & $0.0275(12)$ & $-0.0006(9)$ & $0.0035(9)$ & $-0.0071(9)$ \\
\hline C13 & $0.0279(11)$ & $0.0252(12)$ & $0.0215(10)$ & $0.0042(9)$ & $0.0057(9)$ & $-0.0004(9)$ \\
\hline $\mathrm{C} 14$ & $0.0328(12)$ & $0.0281(12)$ & $0.0231(11)$ & $0.0027(10)$ & $0.0097(9)$ & $-0.0008(9)$ \\
\hline $\mathrm{C} 15$ & $0.0196(10)$ & $0.0251(11)$ & $0.0279(11)$ & $0.0010(9)$ & $-0.0007(8)$ & $-0.0013(9)$ \\
\hline $\mathrm{C} 16$ & $0.0249(11)$ & $0.0302(12)$ & $0.0197(10)$ & $0.0039(10)$ & $0.0012(8)$ & $-0.0003(9)$ \\
\hline $\mathrm{C} 17$ & $0.0223(10)$ & $0.0193(10)$ & $0.0201(10)$ & $0.0020(8)$ & $0.0031(8)$ & $-0.0001(8)$ \\
\hline C18 & $0.0220(10)$ & $0.0184(10)$ & $0.0183(10)$ & $0.0003(8)$ & $0.0030(8)$ & $-0.0018(8)$ \\
\hline C19 & $0.0237(11)$ & $0.0301(12)$ & $0.0185(10)$ & $0.0015(9)$ & $0.0020(8)$ & $0.0010(9)$ \\
\hline $\mathrm{C} 20$ & $0.0214(10)$ & $0.0275(12)$ & $0.0222(10)$ & $0.0025(9)$ & $0.0014(8)$ & $0.0010(9)$ \\
\hline $\mathrm{C} 21$ & $0.0201(10)$ & $0.0202(10)$ & $0.0196(10)$ & $0.0004(8)$ & $0.0018(8)$ & $-0.0010(8)$ \\
\hline $\mathrm{C} 22$ & $0.0282(12)$ & $0.0242(12)$ & $0.0289(12)$ & $-0.0014(9)$ & $0.0077(9)$ & $-0.0052(9)$ \\
\hline $\mathrm{C} 23$ & $0.0218(10)$ & $0.0189(10)$ & $0.0182(10)$ & $0.0024(8)$ & $0.0021(8)$ & $0.0001(8)$ \\
\hline $\mathrm{C} 24$ & $0.0290(12)$ & $0.0309(12)$ & $0.0218(11)$ & $0.0071(10)$ & $0.0040(9)$ & 0.0045 (9) \\
\hline $\mathrm{C} 25$ & $0.0296(12)$ & $0.0307(12)$ & $0.0198(10)$ & $0.0046(10)$ & $0.0046(9)$ & $0.0023(9)$ \\
\hline $\mathrm{C} 26$ & $0.0231(10)$ & $0.0205(10)$ & $0.0197(10)$ & $0.0017(9)$ & $0.0021(8)$ & $-0.0011(8)$ \\
\hline $\mathrm{C} 27$ & $0.0236(11)$ & $0.0284(12)$ & $0.0237(11)$ & $-0.0004(9)$ & $0.0054(8)$ & $-0.0043(9)$ \\
\hline $\mathrm{C} 28$ & $0.0238(12)$ & $0.0484(16)$ & $0.0306(13)$ & $0.0023(11)$ & $0.0035(9)$ & $-0.0017(11)$ \\
\hline $\mathrm{C} 29$ & $0.0250(11)$ & $0.0375(14)$ & $0.0265(12)$ & $0.0007(10)$ & $0.0060(9)$ & $-0.0080(10)$ \\
\hline $\mathrm{C} 30$ & $0.0272(11)$ & $0.0301(12)$ & $0.0252(11)$ & $0.0006(10)$ & $0.0061(9)$ & $-0.0047(10)$ \\
\hline $\mathrm{C} 31$ & $0.0302(13)$ & $0.0496(16)$ & $0.0273(12)$ & $-0.0016(11)$ & $0.0078(10)$ & $-0.0125(11)$ \\
\hline $\mathrm{C} 32$ & $0.0317(12)$ & $0.0334(13)$ & $0.0262(11)$ & $0.0029(10)$ & $0.0095(9)$ & $0.0004(10)$ \\
\hline $\mathrm{C} 33$ & $0.0514(17)$ & $0.0420(16)$ & $0.0376(15)$ & $-0.0039(13)$ & 0.0157 (13) & $-0.0029(12)$ \\
\hline $\mathrm{C} 34$ & $0.0498(18)$ & $0.061(2)$ & 0.0543 (19) & $-0.0098(15)$ & $0.0281(15)$ & $-0.0249(16)$ \\
\hline
\end{tabular}


Geometric parameters $\left(\AA,{ }^{\circ}\right)$

\begin{tabular}{|c|c|c|c|}
\hline $\mathrm{O} 1-\mathrm{C} 7$ & $1.348(3)$ & $\mathrm{C} 18-\mathrm{C} 19$ & $1.534(3)$ \\
\hline $\mathrm{O} 1-\mathrm{C} 8$ & $1.458(2)$ & $\mathrm{C} 18-\mathrm{H} 18$ & 0.9800 \\
\hline $\mathrm{O} 2-\mathrm{C} 7$ & $1.196(3)$ & $\mathrm{C} 19-\mathrm{C} 20$ & $1.539(3)$ \\
\hline $\mathrm{C} 1-\mathrm{C} 2$ & $1.510(4)$ & $\mathrm{C} 19-\mathrm{H} 19 \mathrm{~A}$ & 0.9700 \\
\hline $\mathrm{C} 1-\mathrm{H} 1 \mathrm{~A}$ & 0.9600 & C19-H19B & 0.9700 \\
\hline $\mathrm{C} 1-\mathrm{H} 1 \mathrm{~B}$ & 0.9600 & $\mathrm{C} 20-\mathrm{C} 21$ & $1.536(3)$ \\
\hline $\mathrm{C} 1-\mathrm{H} 1 \mathrm{C}$ & 0.9600 & $\mathrm{C} 20-\mathrm{H} 20 \mathrm{~A}$ & 0.9700 \\
\hline $\mathrm{C} 2-\mathrm{C} 3$ & $1.523(3)$ & $\mathrm{C} 20-\mathrm{H} 20 \mathrm{~B}$ & 0.9700 \\
\hline $\mathrm{C} 2-\mathrm{H} 2 \mathrm{~A}$ & 0.9700 & $\mathrm{C} 21-\mathrm{C} 22$ & $1.530(3)$ \\
\hline $\mathrm{C} 2-\mathrm{H} 2 \mathrm{~B}$ & 0.9700 & $\mathrm{C} 21-\mathrm{C} 23$ & $1.538(3)$ \\
\hline $\mathrm{C} 3-\mathrm{C} 4$ & $1.512(4)$ & $\mathrm{C} 21-\mathrm{C} 26$ & $1.557(3)$ \\
\hline $\mathrm{C} 3-\mathrm{H} 3 \mathrm{~A}$ & 0.9700 & $\mathrm{C} 22-\mathrm{H} 22 \mathrm{~A}$ & 0.9600 \\
\hline $\mathrm{C} 3-\mathrm{H} 3 \mathrm{~B}$ & 0.9700 & $\mathrm{C} 22-\mathrm{H} 22 \mathrm{~B}$ & 0.9600 \\
\hline $\mathrm{C} 4-\mathrm{C} 5$ & $1.530(3)$ & $\mathrm{C} 22-\mathrm{H} 22 \mathrm{C}$ & 0.9600 \\
\hline $\mathrm{C} 4-\mathrm{H} 4 \mathrm{~A}$ & 0.9700 & $\mathrm{C} 23-\mathrm{C} 24$ & $1.527(3)$ \\
\hline $\mathrm{C} 4-\mathrm{H} 4 \mathrm{~B}$ & 0.9700 & $\mathrm{C} 23-\mathrm{H} 23$ & 0.9800 \\
\hline $\mathrm{C} 5-\mathrm{C} 6$ & $1.516(3)$ & $\mathrm{C} 24-\mathrm{C} 25$ & $1.538(3)$ \\
\hline $\mathrm{C} 5-\mathrm{H} 5 \mathrm{~A}$ & 0.9700 & $\mathrm{C} 24-\mathrm{H} 24 \mathrm{~A}$ & 0.9700 \\
\hline C5-H5B & 0.9700 & $\mathrm{C} 24-\mathrm{H} 24 \mathrm{~B}$ & 0.9700 \\
\hline $\mathrm{C} 6-\mathrm{C} 7$ & $1.510(3)$ & $\mathrm{C} 25-\mathrm{C} 26$ & $1.563(3)$ \\
\hline C6-H6A & 0.9700 & $\mathrm{C} 25-\mathrm{H} 25 \mathrm{~A}$ & 0.9700 \\
\hline C6-H6B & 0.9700 & $\mathrm{C} 25-\mathrm{H} 25 \mathrm{~B}$ & 0.9700 \\
\hline $\mathrm{C} 8-\mathrm{C} 14$ & $1.513(3)$ & $\mathrm{C} 26-\mathrm{C} 27$ & $1.535(3)$ \\
\hline $\mathrm{C} 8-\mathrm{C} 9$ & $1.518(3)$ & $\mathrm{C} 26-\mathrm{H} 26$ & 0.9800 \\
\hline $\mathrm{C} 8-\mathrm{H} 8$ & 0.9800 & $\mathrm{C} 27-\mathrm{C} 28$ & $1.528(3)$ \\
\hline $\mathrm{C} 9-\mathrm{C} 10$ & $1.519(3)$ & $\mathrm{C} 27-\mathrm{C} 29$ & $1.539(3)$ \\
\hline C9-H9A & 0.9700 & $\mathrm{C} 27-\mathrm{H} 27$ & 0.9800 \\
\hline C9- & 0.9700 & $\mathrm{C} 28-\mathrm{H} 28 \mathrm{~A}$ & 0.9600 \\
\hline $\mathrm{C} 10-\mathrm{C} 15$ & $1.330(3)$ & $\mathrm{C} 28-\mathrm{H} 28 \mathrm{~B}$ & 0.9600 \\
\hline $\mathrm{C} 10-\mathrm{C} 11$ & $1.520(3)$ & $\mathrm{C} 28-\mathrm{H} 28 \mathrm{C}$ & 0.9600 \\
\hline $\mathrm{C} 11-\mathrm{C} 12$ & $1.545(3)$ & $\mathrm{C} 29-\mathrm{C} 30$ & $1.525(3)$ \\
\hline $\mathrm{C} 11-\mathrm{C} 13$ & $1.549(3)$ & $\mathrm{C} 29-\mathrm{H} 29 \mathrm{~A}$ & 0.9700 \\
\hline $\mathrm{C} 11-\mathrm{C} 18$ & $1.558(3)$ & $\mathrm{C} 29-\mathrm{H} 29 \mathrm{~B}$ & 0.9700 \\
\hline $\mathrm{C} 12-\mathrm{H} 12 \mathrm{~A}$ & 0.9600 & $\mathrm{C} 30-\mathrm{C} 31$ & $1.523(3)$ \\
\hline $\mathrm{C} 12-\mathrm{H} 12 \mathrm{~B}$ & 0.9600 & $\mathrm{C} 30-\mathrm{H} 30 \mathrm{~A}$ & 0.9700 \\
\hline $\mathrm{C} 12-\mathrm{H} 12 \mathrm{C}$ & 0.9600 & $\mathrm{C} 30-\mathrm{H} 30 \mathrm{~B}$ & 0.9700 \\
\hline $\mathrm{C} 13-\mathrm{C} 14$ & $1.526(3)$ & $\mathrm{C} 31-\mathrm{C} 32$ & $1.524(3)$ \\
\hline $\mathrm{C} 13-\mathrm{H} 13 \mathrm{~A}$ & 0.9700 & $\mathrm{C} 31-\mathrm{H} 31 \mathrm{~A}$ & 0.9700 \\
\hline C13-H13B & 0.9700 & $\mathrm{C} 31-\mathrm{H} 31 \mathrm{~B}$ & 0.9700 \\
\hline $\mathrm{C} 14-\mathrm{H} 14 \mathrm{~A}$ & 0.9700 & $\mathrm{C} 32-\mathrm{C} 33$ & $1.509(4)$ \\
\hline C14-H14B & 0.9700 & $\mathrm{C} 32-\mathrm{C} 34$ & $1.518(4)$ \\
\hline $\mathrm{C} 15-\mathrm{C} 16$ & $1.497(3)$ & $\mathrm{C} 32-\mathrm{H} 32$ & 0.9800 \\
\hline C15-H15 & 0.9300 & $\mathrm{C} 33-\mathrm{H} 33 \mathrm{~A}$ & 0.9600 \\
\hline $\mathrm{C} 16-\mathrm{C} 17$ & $1.523(3)$ & $\mathrm{C} 33-\mathrm{H} 33 \mathrm{~B}$ & 0.9600 \\
\hline C16-H16A & 0.9700 & $\mathrm{C} 33-\mathrm{H} 33 \mathrm{C}$ & 0.9600 \\
\hline $\mathrm{C} 16-\mathrm{H} 16 \mathrm{~B}$ & 0.9700 & C34-H34A & 0.9600 \\
\hline
\end{tabular}




\begin{tabular}{|c|c|c|c|}
\hline $\mathrm{C} 17-\mathrm{C} 23$ & $1.524(3)$ & $\mathrm{C} 34-\mathrm{H} 34 \mathrm{~B}$ & 0.9600 \\
\hline $\mathrm{C} 17-\mathrm{C} 18$ & $1.544(3)$ & $\mathrm{C} 34-\mathrm{H} 34 \mathrm{C}$ & 0.9600 \\
\hline $\mathrm{C} 17-\mathrm{H} 17$ & 0.9800 & & \\
\hline $\mathrm{O} 2 \cdots \mathrm{C} 14$ & $3.277(2)$ & $\mathrm{H} 4 \mathrm{~B} \cdots \mathrm{H} 9 \mathrm{~B}^{\mathrm{i}}$ & 2.56 \\
\hline $\mathrm{O} 2 \cdots \mathrm{H} 5 \mathrm{~A}$ & 2.83 & $\mathrm{H} 5 \mathrm{~A} \cdots \mathrm{H} 33 \mathrm{C}^{\mathrm{vii}}$ & 2.45 \\
\hline $\mathrm{O} 2 \cdots \mathrm{H} 5 \mathrm{~B}$ & 2.73 & H5B $\cdots \mathrm{H}_{6} \mathrm{~A}^{\mathrm{i}}$ & 2.51 \\
\hline $\mathrm{O} 2 \cdots \mathrm{H} 8$ & 2.43 & H8 $\cdots \mathrm{H} 13 \mathrm{~B}$ & 2.56 \\
\hline $\mathrm{O} 2 \cdots \mathrm{H} 14 \mathrm{~B}$ & 2.84 & H9A $\cdots \mathrm{H} 15$ & 2.26 \\
\hline $\mathrm{O} 2 \cdots \mathrm{H} 4 \mathrm{~A}^{\mathrm{i}}$ & 2.75 & $\mathrm{H} 9 \mathrm{~A} \cdots \mathrm{H} 28 \mathrm{~A}^{\mathrm{v}}$ & 2.58 \\
\hline $\mathrm{C} 20 \cdots \mathrm{C} 28$ & 3.309 (2) & $\mathrm{H} 9 \mathrm{~B} \cdots \mathrm{H} 12 \mathrm{C}$ & 2.30 \\
\hline $\mathrm{C} 22 \cdots \mathrm{C} 28$ & $3.556(2)$ & H9B $\cdots H 14 A$ & 2.58 \\
\hline C $3 \cdots$ H6B & 2.86 & $\mathrm{H} 12 \mathrm{~A} \cdots \mathrm{H} 13 \mathrm{~A}$ & 2.40 \\
\hline C6 6 -H3A & 2.81 & H12A $\cdots H 19 A$ & 2.21 \\
\hline C7 $\cdots \mathrm{H} 14 \mathrm{~B}$ & 2.97 & H12B $\cdots H 17$ & 2.30 \\
\hline $\mathrm{C} 9 \cdots \mathrm{H} 12 \mathrm{C}$ & 2.78 & $\mathrm{H} 12 \mathrm{C} \cdots \mathrm{H} 14 \mathrm{~A}$ & 2.37 \\
\hline $\mathrm{C} 12 \cdots \mathrm{H} 17$ & 2.90 & H13A $\cdots H 19 B$ & 2.29 \\
\hline $\mathrm{C} 12 \cdots \mathrm{H} 9 \mathrm{~B}$ & 2.92 & $\mathrm{H} 13 \mathrm{~B} \cdots \mathrm{H} 18$ & 2.27 \\
\hline $\mathrm{C} 12 \cdots \mathrm{H} 14 \mathrm{~A}$ & 2.85 & $\mathrm{H} 13 \mathrm{~B} \cdots \mathrm{H} 24 \mathrm{~B}^{\mathrm{vii}}$ & 2.41 \\
\hline 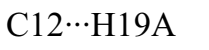 & 2.63 & H14B $\cdots H 34 B^{\text {viii }}$ & 2.58 \\
\hline C13ㄱ19B & 2.79 & $\mathrm{H} 15 \cdots \mathrm{H} 28 \mathrm{~A}^{\mathrm{v}}$ & 2.54 \\
\hline $\mathrm{C} 14 \cdots \mathrm{H} 12 \mathrm{C}$ & 2.87 & $\mathrm{H} 15 \cdots \mathrm{H} 30 \mathrm{~A}^{\mathrm{v}}$ & 2.51 \\
\hline $\mathrm{C} 15 \cdots \mathrm{H} 18$ & 2.95 & $\mathrm{H} 16 \mathrm{~A} \cdots \mathrm{H} 18$ & 2.60 \\
\hline $\mathrm{C} 15 \cdots \mathrm{H} 26^{\mathrm{ii}}$ & 2.98 & $\mathrm{H} 16 \mathrm{~A} \cdots \mathrm{H} 23$ & 2.36 \\
\hline $\mathrm{C} 16 \cdots \mathrm{H} 24 \mathrm{~A}$ & 2.93 & $\mathrm{H} 16 \mathrm{~A} \cdots \mathrm{H} 22 \mathrm{C}^{\mathrm{vii}}$ & 2.56 \\
\hline $\mathrm{C} 17 \cdots \mathrm{H} 12 \mathrm{~B}$ & 2.87 & $\mathrm{H} 16 \mathrm{~B} \cdots \mathrm{H} 20 \mathrm{~A}^{\mathrm{ii}}$ & 2.48 \\
\hline $\mathrm{C} 17 \cdots \mathrm{H} 22 \mathrm{C}$ & 2.78 & $\mathrm{H} 17 \cdots \mathrm{H} 22 \mathrm{C}$ & 2.26 \\
\hline $\mathrm{C} 19 \cdots \mathrm{H} 22 \mathrm{C}$ & 2.74 & $\mathrm{H} 18 \cdots \mathrm{H} 23$ & 2.47 \\
\hline $\mathrm{C} 19 \cdots \mathrm{H} 12 \mathrm{~A}$ & 2.73 & $\mathrm{H} 18 \cdots \mathrm{H} 24 \mathrm{~B}^{\mathrm{vii}}$ & 2.39 \\
\hline $\mathrm{C} 19 \cdots \mathrm{H} 13 \mathrm{~A}$ & 2.84 & $\mathrm{H} 19 \mathrm{~A} \cdots \mathrm{H} 22 \mathrm{C}$ & 2.23 \\
\hline $\mathrm{C} 20 \cdots \mathrm{H} 28 \mathrm{~B}$ & 2.87 & $\mathrm{H} 20 \mathrm{~A} \cdots \mathrm{H} 23$ & 2.39 \\
\hline $\mathrm{C} 21 \cdots \mathrm{H} 28 \mathrm{~B}$ & 2.93 & $\mathrm{H} 20 \mathrm{~A} \cdots \mathrm{H} 26$ & 2.45 \\
\hline $\mathrm{C} 22 \cdots \mathrm{H} 19 \mathrm{~A}$ & 2.77 & $\mathrm{H} 20 \mathrm{~A} \cdots \mathrm{H} 33 \mathrm{~A}^{\mathrm{ix}}$ & 2.37 \\
\hline $\mathrm{C} 22 \cdots \mathrm{H} 24 \mathrm{~B}$ & 2.86 & $\mathrm{H} 20 \mathrm{~B} \cdots \mathrm{H} 22 \mathrm{~A}$ & 2.48 \\
\hline $\mathrm{C} 22 \cdots \mathrm{H} 30 \mathrm{~A}^{\mathrm{iii}}$ & 2.91 & H20B $\cdots H 28 B$ & 2.17 \\
\hline $\mathrm{C} 22 \cdots \mathrm{H} 27$ & 2.70 & $\mathrm{H} 22 \mathrm{~A} \cdots \mathrm{H} 27$ & 2.41 \\
\hline $\mathrm{C} 22 \cdots \mathrm{H} 17$ & 2.82 & $\mathrm{H} 22 \mathrm{~A} \cdots \mathrm{H} 28 \mathrm{~B}$ & 2.42 \\
\hline $\mathrm{C} 24 \cdots \mathrm{H} 16 \mathrm{~B}$ & 2.88 & $\mathrm{H} 22 \mathrm{~A} \cdots \mathrm{H} 30 \mathrm{~A}^{\mathrm{iii}}$ & 2.55 \\
\hline $\mathrm{C} 24 \cdots \mathrm{H} 22 \mathrm{~B}$ & 2.68 & $\mathrm{H} 22 \mathrm{~B} \cdots \mathrm{H} 24 \mathrm{~B}$ & 2.34 \\
\hline $\mathrm{C} 25 \cdots \mathrm{H} 22 \mathrm{~B}$ & 2.71 & $\mathrm{H} 22 \mathrm{~B} \cdots \mathrm{H} 25 \mathrm{~B}$ & 2.52 \\
\hline $\mathrm{C} 25 \cdots \mathrm{H} 29 \mathrm{~A}$ & 2.51 & $\mathrm{H} 22 \mathrm{~B} \cdots \mathrm{H} 27$ & 2.54 \\
\hline $\mathrm{C} 27 \cdots \mathrm{H} 22 \mathrm{~A}$ & 2.83 & $\mathrm{H} 23 \cdots \mathrm{H} 26$ & 2.37 \\
\hline 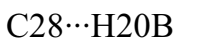 & 2.78 & $\mathrm{H} 25 \mathrm{~A} \cdots \mathrm{H} 29 \mathrm{~A}$ & 2.32 \\
\hline $\mathrm{C} 28 \cdots \mathrm{H} 30 \mathrm{~A}$ & 2.90 & $\mathrm{H} 25 \mathrm{~B} \cdots \mathrm{H} 27$ & 2.45 \\
\hline $\mathrm{C} 29 \cdots \mathrm{H} 25 \mathrm{~B}$ & 2.91 & $\mathrm{H} 25 \mathrm{~B} \cdots \mathrm{H} 29 \mathrm{~A}$ & 2.36 \\
\hline $\mathrm{C} 30 \cdots \mathrm{H} 28 \mathrm{~A}$ & 2.79 & $\mathrm{H} 26 \cdots \mathrm{H} 28 \mathrm{C}$ & 2.50 \\
\hline $\mathrm{C} 30 \cdots \mathrm{H} 33 \mathrm{~A}$ & 2.75 & $\mathrm{H} 27 \cdots \mathrm{H} 30 \mathrm{~B}$ & 2.46 \\
\hline $\mathrm{C} 33 \cdots \mathrm{H} 30 \mathrm{~B}$ & 2.84 & $\mathrm{H} 27 \cdots \mathrm{H} 28 \mathrm{C}^{\mathrm{iii}}$ & 2.53 \\
\hline
\end{tabular}


H1 A $\cdots H 33 B^{\text {iv }}$

H1B $\cdots$ H3A

H1B $\cdots H 34 C^{\text {ii }}$

$\mathrm{H} 1 \mathrm{C} \cdots \mathrm{H} 3 \mathrm{~B}$

H1C $\cdots \mathrm{H}_{13} \mathrm{~A}^{\mathrm{v}}$

$\mathrm{H} 2 \mathrm{~A} \cdots \mathrm{H} 4 \mathrm{~A}$

$\mathrm{H} 2 \mathrm{~A} \cdots \mathrm{H} 14 \mathrm{~B}^{\mathrm{vi}}$

$\mathrm{H} 2 \mathrm{~B} \cdots \mathrm{H} 4 \mathrm{~B}$

$\mathrm{H} 2 \mathrm{~B} \cdots \mathrm{H} 12 \mathrm{C}^{\mathrm{i}}$

H3A $\cdots$ H6B

H3A $\cdots$ H34 ${ }^{\text {ii }}$

$\mathrm{H} 3 \mathrm{~B} \cdots \mathrm{H} 5 \mathrm{~A}$

$\mathrm{H} 4 \mathrm{~A} \cdots \mathrm{H} 6 \mathrm{~A}$

$\mathrm{C} 7-\mathrm{O} 1-\mathrm{C} 8$

$\mathrm{C} 2-\mathrm{C} 1-\mathrm{H} 1 \mathrm{~A}$

$\mathrm{C} 2-\mathrm{C} 1-\mathrm{H} 1 \mathrm{~B}$

$\mathrm{H} 1 \mathrm{~A}-\mathrm{C} 1-\mathrm{H} 1 \mathrm{~B}$

$\mathrm{C} 2-\mathrm{C} 1-\mathrm{H} 1 \mathrm{C}$

$\mathrm{H} 1 \mathrm{~A}-\mathrm{C} 1-\mathrm{H} 1 \mathrm{C}$

$\mathrm{H} 1 \mathrm{~B}-\mathrm{C} 1-\mathrm{H} 1 \mathrm{C}$

$\mathrm{C} 1-\mathrm{C} 2-\mathrm{C} 3$

$\mathrm{C} 1-\mathrm{C} 2-\mathrm{H} 2 \mathrm{~A}$

$\mathrm{C} 3-\mathrm{C} 2-\mathrm{H} 2 \mathrm{~A}$

$\mathrm{C} 1-\mathrm{C} 2-\mathrm{H} 2 \mathrm{~B}$

$\mathrm{C} 3-\mathrm{C} 2-\mathrm{H} 2 \mathrm{~B}$

$\mathrm{H} 2 \mathrm{~A}-\mathrm{C} 2-\mathrm{H} 2 \mathrm{~B}$

$\mathrm{C} 4-\mathrm{C} 3-\mathrm{C} 2$

$\mathrm{C} 4-\mathrm{C} 3-\mathrm{H} 3 \mathrm{~A}$

$\mathrm{C} 2-\mathrm{C} 3-\mathrm{H} 3 \mathrm{~A}$

$\mathrm{C} 4-\mathrm{C} 3-\mathrm{H} 3 \mathrm{~B}$

$\mathrm{C} 2-\mathrm{C} 3-\mathrm{H} 3 \mathrm{~B}$

$\mathrm{H} 3 \mathrm{~A}-\mathrm{C} 3-\mathrm{H} 3 \mathrm{~B}$

$\mathrm{C} 3-\mathrm{C} 4-\mathrm{C} 5$

$\mathrm{C} 3-\mathrm{C} 4-\mathrm{H} 4 \mathrm{~A}$

$\mathrm{C} 5-\mathrm{C} 4-\mathrm{H} 4 \mathrm{~A}$

$\mathrm{C} 3-\mathrm{C} 4-\mathrm{H} 4 \mathrm{~B}$

$\mathrm{C} 5-\mathrm{C} 4-\mathrm{H} 4 \mathrm{~B}$

$\mathrm{H} 4 \mathrm{~A}-\mathrm{C} 4-\mathrm{H} 4 \mathrm{~B}$

$\mathrm{C} 6-\mathrm{C} 5-\mathrm{C} 4$

$\mathrm{C} 6-\mathrm{C} 5-\mathrm{H} 5 \mathrm{~A}$

$\mathrm{C} 4-\mathrm{C} 5-\mathrm{H} 5 \mathrm{~A}$

$\mathrm{C} 6-\mathrm{C} 5-\mathrm{H} 5 \mathrm{~B}$

$\mathrm{C} 4-\mathrm{C} 5-\mathrm{H} 5 \mathrm{~B}$

$\mathrm{H} 5 \mathrm{~A}-\mathrm{C} 5-\mathrm{H} 5 \mathrm{~B}$

$\mathrm{C} 7-\mathrm{C} 6-\mathrm{C} 5$

C7- 6 - $-\mathrm{H} 6 \mathrm{~A}$

$\mathrm{C} 5-\mathrm{C} 6-\mathrm{H} 6 \mathrm{~A}$
2.49

2.55

2.58

2.59

2.51

2.49

2.52

2.55

2.54

2.31

2.52

2.58

2.46

$117.58(19)$

109.5

109.5

109.5

109.5

109.5

109.5

113.9 (2)

108.8

108.8

108.8

108.8

107.7

113.1 (2)

109.0

109.0

109.0

109.0

107.8

$115.3(2)$

108.5

108.5

108.5

108.5

107.5

112.9 (2)

109.0

109.0

109.0

109.0

107.8

113.7 (2)

108.8

108.8
H28A $\cdots H 30 A$

$\mathrm{H} 28 \mathrm{C} \cdots \mathrm{H} 29 \mathrm{~B}$

H29A $\cdots$ H31B

$\mathrm{H} 29 \mathrm{~B} \cdots \mathrm{H} 28 \mathrm{C}$

H29B $\cdots \mathrm{H} 31 \mathrm{~A}$

H30A $\cdots \mathrm{H} 32$

H30B $\cdots$ H33A

H31A $\cdots$ H34A

H31B $\cdots$ H33C

H31B $\cdots$ H34B

H33B $\cdots H 34 C$

H33C $\cdots$ H34B

C17-C18-H18

$\mathrm{C} 11-\mathrm{C} 18-\mathrm{H} 18$

$\mathrm{C} 18-\mathrm{C} 19-\mathrm{C} 20$

C18-C19-H19A

C20-C19-H19A

C18-C19-H19B

C20-C19-H19B

H19A-C19-H19B

$\mathrm{C} 21-\mathrm{C} 20-\mathrm{C} 19$

$\mathrm{C} 21-\mathrm{C} 20-\mathrm{H} 20 \mathrm{~A}$

$\mathrm{C} 19-\mathrm{C} 20-\mathrm{H} 20 \mathrm{~A}$

$\mathrm{C} 21-\mathrm{C} 20-\mathrm{H} 20 \mathrm{~B}$

$\mathrm{C} 19-\mathrm{C} 20-\mathrm{H} 20 \mathrm{~B}$

$\mathrm{H} 20 \mathrm{~A}-\mathrm{C} 20-\mathrm{H} 20 \mathrm{~B}$

$\mathrm{C} 22-\mathrm{C} 21-\mathrm{C} 20$

$\mathrm{C} 22-\mathrm{C} 21-\mathrm{C} 23$

$\mathrm{C} 20-\mathrm{C} 21-\mathrm{C} 23$

$\mathrm{C} 22-\mathrm{C} 21-\mathrm{C} 26$

$\mathrm{C} 20-\mathrm{C} 21-\mathrm{C} 26$

$\mathrm{C} 23-\mathrm{C} 21-\mathrm{C} 26$

$\mathrm{C} 21-\mathrm{C} 22-\mathrm{H} 22 \mathrm{~A}$

$\mathrm{C} 21-\mathrm{C} 22-\mathrm{H} 22 \mathrm{~B}$

$\mathrm{H} 22 \mathrm{~A}-\mathrm{C} 22-\mathrm{H} 22 \mathrm{~B}$

$\mathrm{C} 21-\mathrm{C} 22-\mathrm{H} 22 \mathrm{C}$

$\mathrm{H} 22 \mathrm{~A}-\mathrm{C} 22-\mathrm{H} 22 \mathrm{C}$

$\mathrm{H} 22 \mathrm{~B}-\mathrm{C} 22-\mathrm{H} 22 \mathrm{C}$

C17-C23-C24

$\mathrm{C} 17-\mathrm{C} 23-\mathrm{C} 21$

$\mathrm{C} 24-\mathrm{C} 23-\mathrm{C} 21$

$\mathrm{C} 17-\mathrm{C} 23-\mathrm{H} 23$

$\mathrm{C} 24-\mathrm{C} 23-\mathrm{H} 23$

$\mathrm{C} 21-\mathrm{C} 23-\mathrm{H} 23$

$\mathrm{C} 23-\mathrm{C} 24-\mathrm{C} 25$

C23-C24-H24A
2.26

2.55

2.54

2.55

2.48

2.53

2.33

2.42

2.59

2.52

2.45

2.54

106.3

106.3

$113.82(17)$

108.8

108.8

108.8

108.8

107.7

$111.60(18)$

109.3

109.3

109.3

109.3

108.0

110.77 (18)

$112.56(18)$

$106.26(17)$

$110.19(18)$

$116.73(18)$

99.87 (16)

109.5

109.5

109.5

109.5

109.5

109.5

$118.16(18)$

$115.40(17)$

104.12 (17)

106.1

106.1

106.1

103.79 (18)

111.0 


\begin{tabular}{|c|c|c|c|}
\hline $\mathrm{C} 7-\mathrm{C} 6-\mathrm{H} 6 \mathrm{~B}$ & 108.8 & $\mathrm{C} 25-\mathrm{C} 24-\mathrm{H} 24 \mathrm{~A}$ & 111.0 \\
\hline $\mathrm{C} 5-\mathrm{C} 6-\mathrm{H} 6 \mathrm{~B}$ & 108.8 & $\mathrm{C} 23-\mathrm{C} 24-\mathrm{H} 24 \mathrm{~B}$ & 111.0 \\
\hline $\mathrm{H} 6 \mathrm{~A}-\mathrm{C} 6-\mathrm{H} 6 \mathrm{~B}$ & 107.7 & $\mathrm{C} 25-\mathrm{C} 24-\mathrm{H} 24 \mathrm{~B}$ & 111.0 \\
\hline $\mathrm{O} 2-\mathrm{C} 7-\mathrm{O} 1$ & $123.8(2)$ & $\mathrm{H} 24 \mathrm{~A}-\mathrm{C} 24-\mathrm{H} 24 \mathrm{~B}$ & 109.0 \\
\hline $\mathrm{O} 2-\mathrm{C} 7-\mathrm{C} 6$ & $125.7(2)$ & $\mathrm{C} 24-\mathrm{C} 25-\mathrm{C} 26$ & $106.87(17)$ \\
\hline $\mathrm{O} 1-\mathrm{C} 7-\mathrm{C} 6$ & $110.5(2)$ & $\mathrm{C} 24-\mathrm{C} 25-\mathrm{H} 25 \mathrm{~A}$ & 110.3 \\
\hline $\mathrm{O} 1-\mathrm{C} 8-\mathrm{C} 14$ & $110.61(18)$ & $\mathrm{C} 26-\mathrm{C} 25-\mathrm{H} 25 \mathrm{~A}$ & 110.3 \\
\hline $\mathrm{O} 1-\mathrm{C} 8-\mathrm{C} 9$ & $106.15(18)$ & $\mathrm{C} 24-\mathrm{C} 25-\mathrm{H} 25 \mathrm{~B}$ & 110.3 \\
\hline $\mathrm{C} 14-\mathrm{C} 8-\mathrm{C} 9$ & $110.85(19)$ & $\mathrm{C} 26-\mathrm{C} 25-\mathrm{H} 25 \mathrm{~B}$ & 110.3 \\
\hline $\mathrm{O} 1-\mathrm{C} 8-\mathrm{H} 8$ & 109.7 & $\mathrm{H} 25 \mathrm{~A}-\mathrm{C} 25-\mathrm{H} 25 \mathrm{~B}$ & 108.6 \\
\hline $\mathrm{C} 14-\mathrm{C} 8-\mathrm{H} 8$ & 109.7 & $\mathrm{C} 27-\mathrm{C} 26-\mathrm{C} 21$ & $118.94(17)$ \\
\hline $\mathrm{C} 9-\mathrm{C} 8-\mathrm{H} 8$ & 109.7 & $\mathrm{C} 27-\mathrm{C} 26-\mathrm{C} 25$ & $112.11(18)$ \\
\hline $\mathrm{C} 8-\mathrm{C} 9-\mathrm{C} 10$ & $111.26(19)$ & $\mathrm{C} 21-\mathrm{C} 26-\mathrm{C} 25$ & $103.22(17)$ \\
\hline $\mathrm{C} 8-\mathrm{C} 9-\mathrm{H} 9 \mathrm{~A}$ & 109.4 & $\mathrm{C} 27-\mathrm{C} 26-\mathrm{H} 26$ & 107.3 \\
\hline $\mathrm{C} 10-\mathrm{C} 9-\mathrm{H} 9 \mathrm{~A}$ & 109.4 & $\mathrm{C} 21-\mathrm{C} 26-\mathrm{H} 26$ & 107.3 \\
\hline $\mathrm{C} 8-\mathrm{C} 9-\mathrm{H} 9 \mathrm{~B}$ & 109.4 & $\mathrm{C} 25-\mathrm{C} 26-\mathrm{H} 26$ & 107.3 \\
\hline $\mathrm{C} 10-\mathrm{C} 9-\mathrm{H} 9 \mathrm{~B}$ & 109.4 & $\mathrm{C} 28-\mathrm{C} 27-\mathrm{C} 26$ & $112.24(18)$ \\
\hline $\mathrm{H} 9 \mathrm{~A}-\mathrm{C} 9-\mathrm{H} 9 \mathrm{~B}$ & 108.0 & $\mathrm{C} 28-\mathrm{C} 27-\mathrm{C} 29$ & $110.25(19)$ \\
\hline $\mathrm{C} 15-\mathrm{C} 10-\mathrm{C} 9$ & $120.0(2)$ & $\mathrm{C} 26-\mathrm{C} 27-\mathrm{C} 29$ & $110.60(18)$ \\
\hline $\mathrm{C} 15-\mathrm{C} 10-\mathrm{C} 11$ & $123.19(19)$ & $\mathrm{C} 28-\mathrm{C} 27-\mathrm{H} 27$ & 107.9 \\
\hline $\mathrm{C} 9-\mathrm{C} 10-\mathrm{C} 11$ & $116.77(18)$ & $\mathrm{C} 26-\mathrm{C} 27-\mathrm{H} 27$ & 107.9 \\
\hline $\mathrm{C} 10-\mathrm{C} 11-\mathrm{C} 12$ & $108.74(18)$ & $\mathrm{C} 29-\mathrm{C} 27-\mathrm{H} 27$ & 107.9 \\
\hline $\mathrm{C} 10-\mathrm{C} 11-\mathrm{C} 13$ & $108.31(17)$ & $\mathrm{C} 27-\mathrm{C} 28-\mathrm{H} 28 \mathrm{~A}$ & 109.5 \\
\hline $\mathrm{C} 12-\mathrm{C} 11-\mathrm{C} 13$ & $109.31(18)$ & $\mathrm{C} 27-\mathrm{C} 28-\mathrm{H} 28 \mathrm{~B}$ & 109.5 \\
\hline $\mathrm{C} 10-\mathrm{C} 11-\mathrm{C} 18$ & $110.47(17)$ & $\mathrm{H} 28 \mathrm{~A}-\mathrm{C} 28-\mathrm{H} 28 \mathrm{~B}$ & 109.5 \\
\hline $\mathrm{C} 12-\mathrm{C} 11-\mathrm{C} 18$ & $111.26(18)$ & $\mathrm{C} 27-\mathrm{C} 28-\mathrm{H} 28 \mathrm{C}$ & 109.5 \\
\hline $\mathrm{C} 13-\mathrm{C} 11-\mathrm{C} 18$ & $108.70(17)$ & $\mathrm{H} 28 \mathrm{~A}-\mathrm{C} 28-\mathrm{H} 28 \mathrm{C}$ & 109.5 \\
\hline $\mathrm{C} 11-\mathrm{C} 12-\mathrm{H} 12 \mathrm{~A}$ & 109.5 & $\mathrm{H} 28 \mathrm{~B}-\mathrm{C} 28-\mathrm{H} 28 \mathrm{C}$ & 109.5 \\
\hline $\mathrm{C} 11-\mathrm{C} 12-\mathrm{H} 12 \mathrm{~B}$ & 109.5 & $\mathrm{C} 30-\mathrm{C} 29-\mathrm{C} 27$ & $114.8(2)$ \\
\hline $\mathrm{H} 12 \mathrm{~A}-\mathrm{C} 12-\mathrm{H} 12 \mathrm{~B}$ & 109.5 & $\mathrm{C} 30-\mathrm{C} 29-\mathrm{H} 29 \mathrm{~A}$ & 108.6 \\
\hline $\mathrm{C} 11-\mathrm{C} 12-\mathrm{H} 12 \mathrm{C}$ & 109.5 & $\mathrm{C} 27-\mathrm{C} 29-\mathrm{H} 29 \mathrm{~A}$ & 108.6 \\
\hline $\mathrm{H} 12 \mathrm{~A}-\mathrm{C} 12-\mathrm{H} 12 \mathrm{C}$ & 109.5 & $\mathrm{C} 30-\mathrm{C} 29-\mathrm{H} 29 \mathrm{~B}$ & 108.6 \\
\hline $\mathrm{H} 12 \mathrm{~B}-\mathrm{C} 12-\mathrm{H} 12 \mathrm{C}$ & 109.5 & $\mathrm{C} 27-\mathrm{C} 29-\mathrm{H} 29 \mathrm{~B}$ & 108.6 \\
\hline $\mathrm{C} 14-\mathrm{C} 13-\mathrm{C} 11$ & $114.64(18)$ & $\mathrm{H} 29 \mathrm{~A}-\mathrm{C} 29-\mathrm{H} 29 \mathrm{~B}$ & 107.5 \\
\hline $\mathrm{C} 14-\mathrm{C} 13-\mathrm{H} 13 \mathrm{~A}$ & 108.6 & $\mathrm{C} 31-\mathrm{C} 30-\mathrm{C} 29$ & $112.0(2)$ \\
\hline $\mathrm{C} 11-\mathrm{C} 13-\mathrm{H} 13 \mathrm{~A}$ & 108.6 & $\mathrm{C} 31-\mathrm{C} 30-\mathrm{H} 30 \mathrm{~A}$ & 109.2 \\
\hline $\mathrm{C} 14-\mathrm{C} 13-\mathrm{H} 13 \mathrm{~B}$ & 108.6 & $\mathrm{C} 29-\mathrm{C} 30-\mathrm{H} 30 \mathrm{~A}$ & 109.2 \\
\hline $\mathrm{C} 11-\mathrm{C} 13-\mathrm{H} 13 \mathrm{~B}$ & 108.6 & $\mathrm{C} 31-\mathrm{C} 30-\mathrm{H} 30 \mathrm{~B}$ & 109.2 \\
\hline $\mathrm{H} 13 \mathrm{~A}-\mathrm{C} 13-\mathrm{H} 13 \mathrm{~B}$ & 107.6 & $\mathrm{C} 29-\mathrm{C} 30-\mathrm{H} 30 \mathrm{~B}$ & 109.2 \\
\hline $\mathrm{C} 8-\mathrm{C} 14-\mathrm{C} 13$ & $110.22(18)$ & $\mathrm{H} 30 \mathrm{~A}-\mathrm{C} 30-\mathrm{H} 30 \mathrm{~B}$ & 107.9 \\
\hline $\mathrm{C} 8-\mathrm{C} 14-\mathrm{H} 14 \mathrm{~A}$ & 109.6 & $\mathrm{C} 30-\mathrm{C} 31-\mathrm{C} 32$ & $115.3(2)$ \\
\hline $\mathrm{C} 13-\mathrm{C} 14-\mathrm{H} 14 \mathrm{~A}$ & 109.6 & $\mathrm{C} 30-\mathrm{C} 31-\mathrm{H} 31 \mathrm{~A}$ & 108.5 \\
\hline $\mathrm{C} 8-\mathrm{C} 14-\mathrm{H} 14 \mathrm{~B}$ & 109.6 & $\mathrm{C} 32-\mathrm{C} 31-\mathrm{H} 31 \mathrm{~A}$ & 108.5 \\
\hline $\mathrm{C} 13-\mathrm{C} 14-\mathrm{H} 14 \mathrm{~B}$ & 109.6 & $\mathrm{C} 30-\mathrm{C} 31-\mathrm{H} 31 \mathrm{~B}$ & 108.5 \\
\hline $\mathrm{H} 14 \mathrm{~A}-\mathrm{C} 14-\mathrm{H} 14 \mathrm{~B}$ & 108.1 & $\mathrm{C} 32-\mathrm{C} 31-\mathrm{H} 31 \mathrm{~B}$ & 108.5 \\
\hline $\mathrm{C} 10-\mathrm{C} 15-\mathrm{C} 16$ & $124.8(2)$ & $\mathrm{H} 31 \mathrm{~A}-\mathrm{C} 31-\mathrm{H} 31 \mathrm{~B}$ & 107.5 \\
\hline $\mathrm{C} 10-\mathrm{C} 15-\mathrm{H} 15$ & 117.6 & $\mathrm{C} 33-\mathrm{C} 32-\mathrm{C} 34$ & $110.4(2)$ \\
\hline $\mathrm{C} 16-\mathrm{C} 15-\mathrm{H} 15$ & 117.6 & $\mathrm{C} 33-\mathrm{C} 32-\mathrm{C} 31$ & $113.3(2)$ \\
\hline
\end{tabular}




\begin{tabular}{|c|c|c|c|}
\hline $\mathrm{C} 15-\mathrm{C} 16-\mathrm{C} 17$ & 112.80 & $\mathrm{C} 34-\mathrm{C} 32-\mathrm{C} 31$ & $110.2(2)$ \\
\hline $\mathrm{C} 15-\mathrm{C} 16-\mathrm{H} 16 \mathrm{~A}$ & 109.0 & $\mathrm{C} 33-\mathrm{C} 32-\mathrm{H} 32$ & 107.6 \\
\hline $\mathrm{C} 17-\mathrm{C} 16-\mathrm{H} 16 \mathrm{~A}$ & 109.0 & $\mathrm{C} 34-\mathrm{C} 32-\mathrm{H} 32$ & 107.6 \\
\hline $\mathrm{C} 15-\mathrm{C} 16-\mathrm{H} 16 \mathrm{~B}$ & 109.0 & $\mathrm{C} 31-\mathrm{C} 32-\mathrm{H} 32$ & 107.6 \\
\hline $\mathrm{C} 17-\mathrm{C} 16-\mathrm{H} 16 \mathrm{~B}$ & 109.0 & $\mathrm{C} 32-\mathrm{C} 33-\mathrm{H} 33 \mathrm{~A}$ & 109.5 \\
\hline $\mathrm{H} 16 \mathrm{~A}-\mathrm{C} 16-\mathrm{H} 16 \mathrm{~B}$ & 107.8 & $\mathrm{C} 32-\mathrm{C} 33-\mathrm{H} 33 \mathrm{~B}$ & 109.5 \\
\hline $\mathrm{C} 16-\mathrm{C} 17-\mathrm{C} 23$ & $110.24(17)$ & $\mathrm{H} 33 \mathrm{~A}-\mathrm{C} 33-\mathrm{H} 33 \mathrm{~B}$ & 109.5 \\
\hline $\mathrm{C} 16-\mathrm{C} 17-\mathrm{C} 18$ & $110.06(17)$ & $\mathrm{C} 32-\mathrm{C} 33-\mathrm{H} 33 \mathrm{C}$ & 109.5 \\
\hline $\mathrm{C} 23-\mathrm{C} 17-\mathrm{C} 18$ & $109.77(17)$ & $\mathrm{H} 33 \mathrm{~A}-\mathrm{C} 33-\mathrm{H} 33 \mathrm{C}$ & 109.5 \\
\hline $\mathrm{C} 16-\mathrm{C} 17-\mathrm{H} 17$ & 108.9 & $\mathrm{H} 33 \mathrm{~B}-\mathrm{C} 33-\mathrm{H} 33 \mathrm{C}$ & 109.5 \\
\hline $\mathrm{C} 23-\mathrm{C} 17-\mathrm{H} 17$ & 108.9 & $\mathrm{C} 32-\mathrm{C} 34-\mathrm{H} 34 \mathrm{~A}$ & 109.5 \\
\hline $\mathrm{C} 18-\mathrm{C} 17-\mathrm{H} 17$ & 108.9 & $\mathrm{C} 32-\mathrm{C} 34-\mathrm{H} 34 \mathrm{~B}$ & 109.5 \\
\hline $\mathrm{C} 19-\mathrm{C} 18-\mathrm{C} 17$ & $111.67(17)$ & $\mathrm{H} 34 \mathrm{~A}-\mathrm{C} 34-\mathrm{H} 34 \mathrm{~B}$ & 109.5 \\
\hline $\mathrm{C} 19-\mathrm{C} 18-\mathrm{C} 11$ & $113.81(17)$ & $\mathrm{C} 32-\mathrm{C} 34-\mathrm{H} 34 \mathrm{C}$ & 109.5 \\
\hline $\mathrm{C} 17-\mathrm{C} 18-\mathrm{C} 11$ & $111.91(17)$ & $\mathrm{H} 34 \mathrm{~A}-\mathrm{C} 34-\mathrm{H} 34 \mathrm{C}$ & 109.5 \\
\hline $\mathrm{C} 19-\mathrm{C} 18-\mathrm{H} 18$ & 106.3 & $\mathrm{H} 34 \mathrm{~B}-\mathrm{C} 34-\mathrm{H} 34 \mathrm{C}$ & 109.5 \\
\hline $\mathrm{C} 1-\mathrm{C} 2-\mathrm{C} 3-\mathrm{C} 4$ & $-177.7(2)$ & $\mathrm{C} 12-\mathrm{C} 11-\mathrm{C} 18-\mathrm{C} 17$ & $76.1(2)$ \\
\hline $\mathrm{C} 2-\mathrm{C} 3-\mathrm{C} 4-\mathrm{C} 5$ & $178.5(2)$ & $\mathrm{C} 13-\mathrm{C} 11-\mathrm{C} 18-\mathrm{C} 17$ & $-163.47(18)$ \\
\hline $\mathrm{C} 3-\mathrm{C} 4-\mathrm{C} 5-\mathrm{C} 6$ & $-65.4(3)$ & $\mathrm{C} 17-\mathrm{C} 18-\mathrm{C} 19-\mathrm{C} 20$ & $50.5(2)$ \\
\hline $\mathrm{C} 4-\mathrm{C} 5-\mathrm{C} 6-\mathrm{C} 7$ & $-173.1(2)$ & $\mathrm{C} 11-\mathrm{C} 18-\mathrm{C} 19-\mathrm{C} 20$ & $178.42(19)$ \\
\hline $\mathrm{C} 8-\mathrm{O} 1-\mathrm{C} 7-\mathrm{O} 2$ & $0.5(4)$ & $\mathrm{C} 18-\mathrm{C} 19-\mathrm{C} 20-\mathrm{C} 21$ & $-55.4(3)$ \\
\hline $\mathrm{C} 8-\mathrm{O} 1-\mathrm{C} 7-\mathrm{C} 6$ & $-179.5(2)$ & $\mathrm{C} 19-\mathrm{C} 20-\mathrm{C} 21-\mathrm{C} 22$ & $-66.0(2)$ \\
\hline $\mathrm{C} 5-\mathrm{C} 6-\mathrm{C} 7-\mathrm{O} 2$ & $-5.8(4)$ & $\mathrm{C} 19-\mathrm{C} 20-\mathrm{C} 21-\mathrm{C} 23$ & $56.5(2)$ \\
\hline $\mathrm{C} 5-\mathrm{C} 6-\mathrm{C} 7-\mathrm{O} 1$ & $174.2(2)$ & $\mathrm{C} 19-\mathrm{C} 20-\mathrm{C} 21-\mathrm{C} 26$ & $166.82(18)$ \\
\hline $\mathrm{C} 7-\mathrm{O} 1-\mathrm{C} 8-\mathrm{C} 14$ & $85.5(3)$ & $\mathrm{C} 16-\mathrm{C} 17-\mathrm{C} 23-\mathrm{C} 24$ & $-57.6(3)$ \\
\hline $\mathrm{C} 7-\mathrm{O} 1-\mathrm{C} 8-\mathrm{C} 9$ & $-154.2(2)$ & $\mathrm{C} 18-\mathrm{C} 17-\mathrm{C} 23-\mathrm{C} 24$ & $-179.04(19)$ \\
\hline $\mathrm{O} 1-\mathrm{C} 8-\mathrm{C} 9-\mathrm{C} 10$ & $-175.13(18)$ & $\mathrm{C} 16-\mathrm{C} 17-\mathrm{C} 23-\mathrm{C} 21$ & $178.27(18)$ \\
\hline $\mathrm{C} 14-\mathrm{C} 8-\mathrm{C} 9-\mathrm{C} 10$ & $-55.0(2)$ & $\mathrm{C} 18-\mathrm{C} 17-\mathrm{C} 23-\mathrm{C} 21$ & $56.9(2)$ \\
\hline $\mathrm{C} 8-\mathrm{C} 9-\mathrm{C} 10-\mathrm{C} 15$ & $-129.1(2)$ & $\mathrm{C} 22-\mathrm{C} 21-\mathrm{C} 23-\mathrm{C} 17$ & $61.6(2)$ \\
\hline $\mathrm{C} 8-\mathrm{C} 9-\mathrm{C} 10-\mathrm{C} 11$ & $51.9(3)$ & $\mathrm{C} 20-\mathrm{C} 21-\mathrm{C} 23-\mathrm{C} 17$ & $-59.8(2)$ \\
\hline $\mathrm{C} 15-\mathrm{C} 10-\mathrm{C} 11-\mathrm{C} 12$ & $-107.8(2)$ & $\mathrm{C} 26-\mathrm{C} 21-\mathrm{C} 23-\mathrm{C} 17$ & $178.40(18)$ \\
\hline $\mathrm{C} 9-\mathrm{C} 10-\mathrm{C} 11-\mathrm{C} 12$ & $71.2(2)$ & $\mathrm{C} 22-\mathrm{C} 21-\mathrm{C} 23-\mathrm{C} 24$ & $-69.6(2)$ \\
\hline $\mathrm{C} 15-\mathrm{C} 10-\mathrm{C} 11-\mathrm{C} 13$ & $133.5(2)$ & $\mathrm{C} 20-\mathrm{C} 21-\mathrm{C} 23-\mathrm{C} 24$ & $168.99(18)$ \\
\hline $\mathrm{C} 9-\mathrm{C} 10-\mathrm{C} 11-\mathrm{C} 13$ & $-47.5(2)$ & $\mathrm{C} 26-\mathrm{C} 21-\mathrm{C} 23-\mathrm{C} 24$ & $47.2(2)$ \\
\hline $\mathrm{C} 15-\mathrm{C} 10-\mathrm{C} 11-\mathrm{C} 18$ & $14.6(3)$ & $\mathrm{C} 17-\mathrm{C} 23-\mathrm{C} 24-\mathrm{C} 25$ & $-165.15(19)$ \\
\hline $\mathrm{C} 9-\mathrm{C} 10-\mathrm{C} 11-\mathrm{C} 18$ & $-166.45(19)$ & $\mathrm{C} 21-\mathrm{C} 23-\mathrm{C} 24-\mathrm{C} 25$ & $-35.6(2)$ \\
\hline $\mathrm{C} 10-\mathrm{C} 11-\mathrm{C} 13-\mathrm{C} 14$ & $49.6(2)$ & $\mathrm{C} 23-\mathrm{C} 24-\mathrm{C} 25-\mathrm{C} 26$ & $9.7(3)$ \\
\hline $\mathrm{C} 12-\mathrm{C} 11-\mathrm{C} 13-\mathrm{C} 14$ & $-68.7(2)$ & $\mathrm{C} 22-\mathrm{C} 21-\mathrm{C} 26-\mathrm{C} 27$ & $-46.1(3)$ \\
\hline $\mathrm{C} 18-\mathrm{C} 11-\mathrm{C} 13-\mathrm{C} 14$ & $169.67(18)$ & $\mathrm{C} 20-\mathrm{C} 21-\mathrm{C} 26-\mathrm{C} 27$ & $81.3(3)$ \\
\hline $\mathrm{O} 1-\mathrm{C} 8-\mathrm{C} 14-\mathrm{C} 13$ & $175.0(2)$ & $\mathrm{C} 23-\mathrm{C} 21-\mathrm{C} 26-\mathrm{C} 27$ & $-164.72(19)$ \\
\hline $\mathrm{C} 9-\mathrm{C} 8-\mathrm{C} 14-\mathrm{C} 13$ & $57.6(2)$ & $\mathrm{C} 22-\mathrm{C} 21-\mathrm{C} 26-\mathrm{C} 25$ & $78.7(2)$ \\
\hline $\mathrm{C} 11-\mathrm{C} 13-\mathrm{C} 14-\mathrm{C} 8$ & $-56.5(3)$ & $\mathrm{C} 20-\mathrm{C} 21-\mathrm{C} 26-\mathrm{C} 25$ & $-153.80(19)$ \\
\hline $\mathrm{C} 9-\mathrm{C} 10-\mathrm{C} 15-\mathrm{C} 16$ & $-177.8(2)$ & $\mathrm{C} 23-\mathrm{C} 21-\mathrm{C} 26-\mathrm{C} 25$ & $-39.9(2)$ \\
\hline $\mathrm{C} 11-\mathrm{C} 10-\mathrm{C} 15-\mathrm{C} 16$ & $1.2(4)$ & $\mathrm{C} 24-\mathrm{C} 25-\mathrm{C} 26-\mathrm{C} 27$ & $148.31(19)$ \\
\hline $\mathrm{C} 10-\mathrm{C} 15-\mathrm{C} 16-\mathrm{C} 17$ & $13.7(3)$ & $\mathrm{C} 24-\mathrm{C} 25-\mathrm{C} 26-\mathrm{C} 21$ & $19.1(2)$ \\
\hline $\mathrm{C} 15-\mathrm{C} 16-\mathrm{C} 17-\mathrm{C} 23$ & $-164.41(19)$ & $\mathrm{C} 21-\mathrm{C} 26-\mathrm{C} 27-\mathrm{C} 28$ & $-59.3(3)$ \\
\hline $\mathrm{C} 15-\mathrm{C} 16-\mathrm{C} 17-\mathrm{C} 18$ & $-43.2(2)$ & $\mathrm{C} 25-\mathrm{C} 26-\mathrm{C} 27-\mathrm{C} 28$ & $-179.7(2)$ \\
\hline
\end{tabular}




$\begin{array}{llll}\mathrm{C} 16-\mathrm{C} 17-\mathrm{C} 18-\mathrm{C} 19 & -170.74(18) & \mathrm{C} 21-\mathrm{C} 26-\mathrm{C} 27-\mathrm{C} 29 & 177.2(2) \\ \mathrm{C} 23-\mathrm{C} 17-\mathrm{C} 18-\mathrm{C} 19 & -49.2(2) & \mathrm{C} 25-\mathrm{C} 26-\mathrm{C} 27-\mathrm{C} 29 & 56.7(3) \\ \mathrm{C} 16-\mathrm{C} 17-\mathrm{C} 18-\mathrm{C} 11 & 60.4(2) & \mathrm{C} 28-\mathrm{C} 27-\mathrm{C} 29-\mathrm{C} 30 & 72.1(3) \\ \mathrm{C} 23-\mathrm{C} 17-\mathrm{C} 18-\mathrm{C} 11 & -178.14(17) & \mathrm{C} 26-\mathrm{C} 27-\mathrm{C} 29-\mathrm{C} 30 & -163.2(2) \\ \mathrm{C} 10-\mathrm{C} 11-\mathrm{C} 18-\mathrm{C} 19 & -172.54(18) & \mathrm{C} 27-\mathrm{C} 29-\mathrm{C} 30-\mathrm{C} 31 & 174.7(2) \\ \mathrm{C} 12-\mathrm{C} 11-\mathrm{C} 18-\mathrm{C} 19 & -51.7(2) & \mathrm{C} 29-\mathrm{C} 30-\mathrm{C} 31-\mathrm{C} 32 & 170.8(2) \\ \mathrm{C} 13-\mathrm{C} 11-\mathrm{C} 18-\mathrm{C} 19 & 68.8(2) & \mathrm{C} 30-\mathrm{C} 31-\mathrm{C} 32-\mathrm{C} 33 & 58.8(3) \\ \mathrm{C} 10-\mathrm{C} 11-\mathrm{C} 18-\mathrm{C} 17 & -44.8(2) & \mathrm{C} 30-\mathrm{C} 31-\mathrm{C} 32-\mathrm{C} 34 & -176.9(3)\end{array}$

Symmetry codes: (i) $-x+2, y+1 / 2,-z$; (ii) $-x+1, y-1 / 2,-z+1$; (iii) $-x, y-1 / 2,-z+1$; (iv) $x+2, y, z-1$; (v) $x+1, y, z$; (vi) $-x+2, y-1 / 2,-z$; (vii) $-x+1, y+1 / 2$, $-z+1$; (viii) $x+1, y, z-1$; (ix) $-x, y+1 / 2,-z+1$. 